DESY 20-170

\title{
A hybrid simulation of gravitational wave production in first-order phase transitions
}

\author{
Ryusuke Jinno, Thomas Konstandin and Henrique Rubira \\ Deutsches Elektronen-Synchrotron DESY, 22607 Hamburg, Germany
}

\begin{abstract}
The LISA telescope will provide the first opportunity to probe the scenario of a first-order phase transition happening close to the electroweak scale. By now, it is evident that the main contribution to the GW spectrum comes from the sound waves propagating through the plasma. Current estimates of the GW spectrum are based on numerical simulations of a scalar field interacting with the plasma or on analytical approximations - the so-called sound shell model. In this work we present a novel setup to calculate the GW spectra from sound waves. We use a hybrid method that uses a 1d simulation (with spherical symmetry) to evolve the velocity and enthalpy profiles of a single bubble after collision and embed it in a $3 \mathrm{~d}$ realization of multiple bubble collisions, assuming linear superposition of the velocity and enthalpy. The main advantage of our method compared to $3 \mathrm{~d}$ hydrodynamic simulations is that it does not require to resolve the scale of bubble wall thickness. This makes our simulations more economical and the only two relevant physical length scales that enter are the bubble size and the shell thickness (that are in turn enclosed by the box size and the grid spacing). The reduced costs allow for extensive parameter studies and we provide a parametrization of the final GW spectrum as a function of the wall velocity and the fluid kinetic energy.
\end{abstract}




\section{Contents}

1 Introduction $\quad 1$

2 Strategy 2

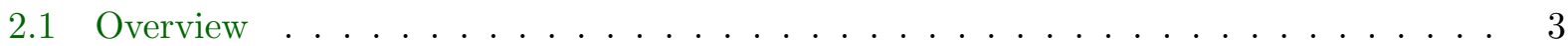

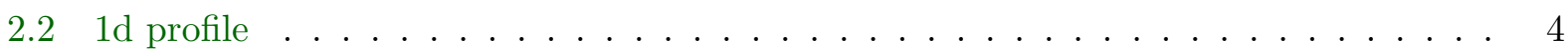

2.2.1 Equations, initial conditions, and shocks . . . . . . . . . . . . . 4

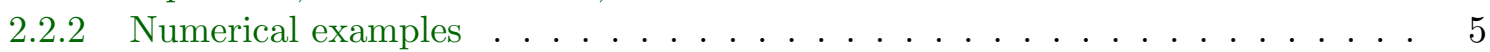

2.33 d embedding . . . . . . . . . . . . . . . . . . . . . . 6

$\begin{array}{lll}3 & \text { Main results } & \mathbf{7}\end{array}$

3.1 Example of the $\mathrm{GW}$ spectrum . . . . . . . . . . . . . . . . 7

3.2 Extraction of the linear growth of the spectrum . . . . . . . . . . . . 9

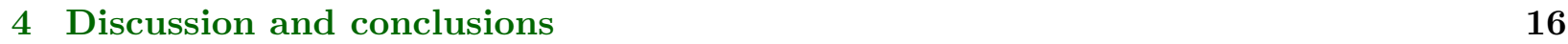

$\begin{array}{ll}\text { A Numerical Evolution of the 1d profile } & 17\end{array}$

A.1 Equations . . . . . . . . . . . . . . . . . . . 17

A.2 Numerical schemes . . . . . . . . . . . . . . . . . . . 18

B From the 3d velocity grid to the GW spectra $\quad 20$

C The first collision of the fluid $\quad 22$

D Normalization of the spectrum $\quad 23$

$\begin{array}{lll}\text { E Fitting } & 23\end{array}$ 


\section{Introduction}

LIGO's milestone discovery of gravitational waves (GW) from Black Hole and Neutron Star [1-3] binaries has opened a new avenue for testing and eventually finding new physics, using gravitational waves science to probe phenomena from microscopic to cosmological scales. Moreover, the upcoming LISA launch $[4,5]$, planned to happen in the mid-2030s is especially interesting for scrutinizing gravitational waves sourced by first-order phase transitions around the $\mathrm{TeV}$ scale.

Even though within the Standard Model (SM) the Higgs field performs a cross-over from the false to the true vacuum [6-8], in many extensions of the SM this scenario is modified to be a first-order phase transition. Further motivation for this scenario is the possibility of explaining the baryon asymmetry through sphaleron processes in electroweak baryogenesis [9-13]. When the Universe cools down and an energetic barrier in the Higgs potential separating the true and the false vacua arises, there is a chance that disjoint regions of the space perform the transition from the symmetric vacuum to the broken one either via quantum or thermal processes [14-16]. Those true vacuum regions grow as spherical bubbles and eventually collide, breaking up spherical symmetry and generating a quadrupole moment and therefore gravitational waves [17].

In recent years, a large effort has been made to better understand the bubble expansion dynamics. This depends on a balance between a driving term proportional to the energy difference between the two vacua and a friction term proportional to the interactions of the scalar field with the surrounding plasma [18]. Next-to-leading order calculations of the friction term have shown that it is hard for bubbles to runaway [19], as initially argued by Ref. [20] (see Ref. [21] for recent developments). Macroscopically, the parameter $\alpha$, the ratio between the energy released in the phase transition and the initial plasma energy density before transition, characterize the bubble dynamics ${ }^{1}$. It basically quantifies how much energy is available to be converted into shear stress through the interaction of the scalar field and the fluid. In the regime of weak phase transitions $(\alpha \ll 1)$, less energy is released to the fluid sector, such that the system is rather linear (meaning smaller velocities) and the fluid is well-characterized by overlapping sound-waves.

Currently, the only simulations of a system including a relativistic fluid and a scalar field are performed by the Helsinki-Sussex group [23-25]. These simulations require on the one hand a simulation volume large enough to fit a sizable number of bubbles (at least 100s). On the other hand, the grid spacing must be small enough to resolve the Higgs bubble wall thickness. The wall velocity in these simulations is adjusted by adding a phenomenological friction term to the Higgs equation. These results are then extrapolated to the physical point, where the Higgs wall thickness is many orders smaller than the bubble size.

In this work we present a new method to calculate the sound shell contribution of GWs. The main intent of our approach is to remove the Higgs bubble wall thickness as a relevant scale from the simulation. The only physically relevant scales in our simulation are then the bubble size and the fluid shell thickness that depending on the wall velocity and the strength of the phase transition is somewhat smaller than the bubble size. Our simulations then require to have a volume large enough to fit a sizable number of bubbles (for enough statistics and to resolve the IR tail of the spectrum) and a grid spacing that is fine enough to resolve the shell thickness. Unlike the full hydrodynamic simulations, a moderate grid size of $N^{3}=256^{3}$ typically suffices to meet these demands. Besides, our approach can cope with rather large time steps in the simulation. In essence, this allows us to run a large suit of simulations and provide the spectrum for a large range of wall velocities and different phase transition strengths. We can also use realistic bubble nucleation histories with an exponential increase in nucleation probability. In Fig. 1 we show an example slice of the fluid in

\footnotetext{
${ }^{1}$ For a recent analysis on the energy budget of phase transitions see Ref. [22].
} 
one of our numerical simulations. We explain the setup of our simulation in Section 2. The main results are shown in Section 3 and we conclude in Section 4.
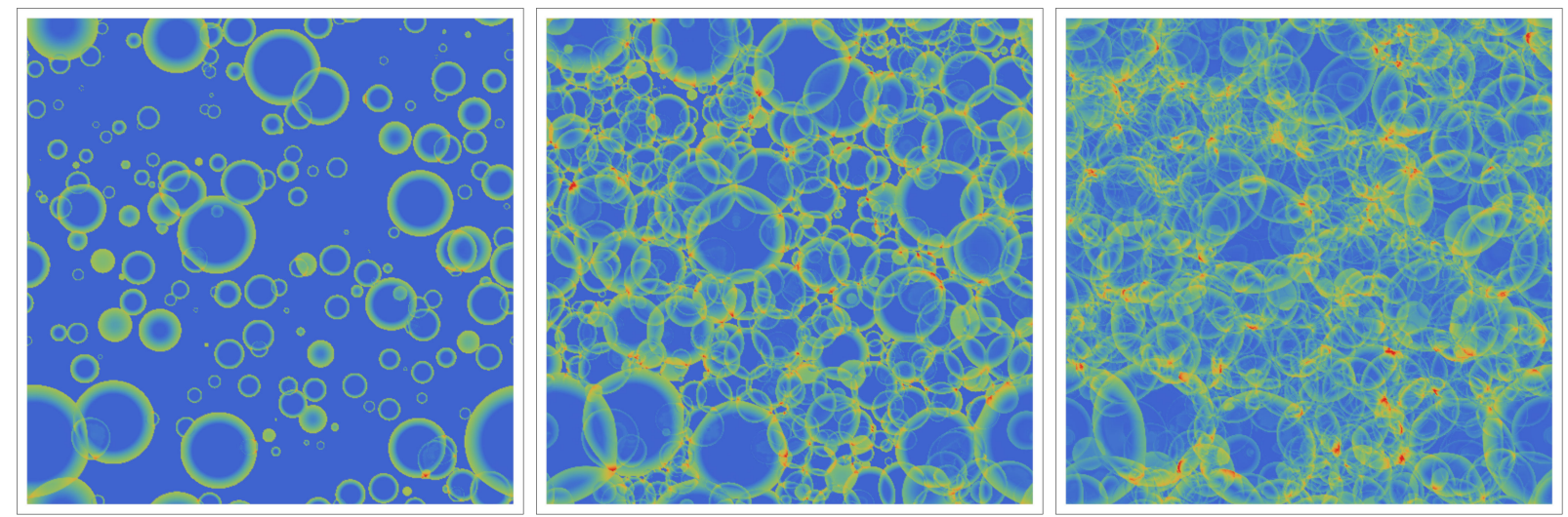

Figure 1: An example slice of the numerical simulation. In this figure we used $\xi_{w}=0.8$ and $v_{\max }=0.1$ and the box size $V=L^{3}$ with $L=80 \xi_{w} / \beta=64 / \beta$ and the grid size $N^{3}=512^{3}$.

$3 d$ simulation

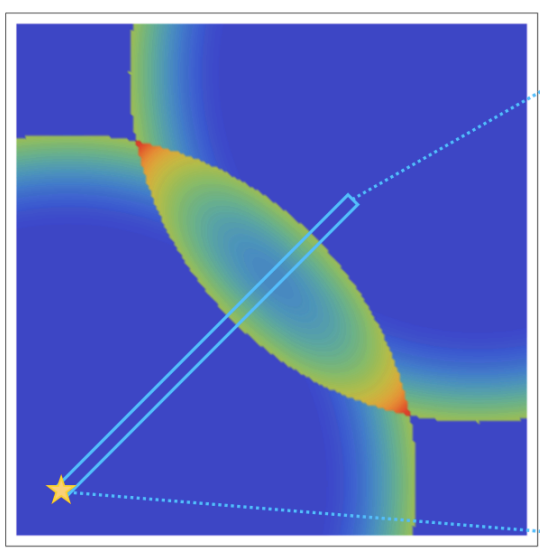

$1 d$ simulation

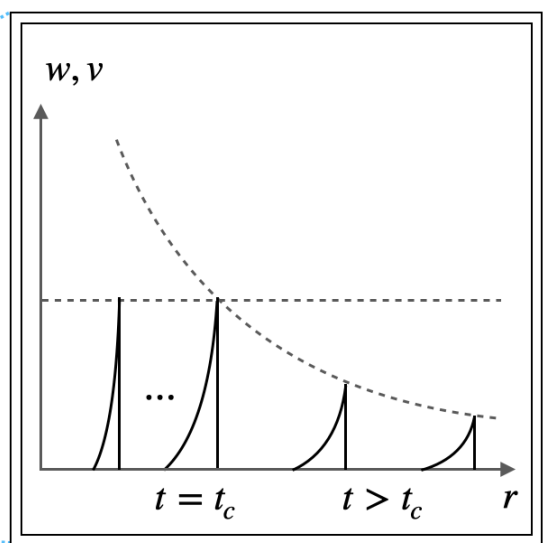

Figure 2: Schematic illustration for the numerical simulation. In the 3d simulation we generate bubble nucleation points (denoted by the star) numerically. For each direction we embed the $1 \mathrm{~d}$ fluid profile with direction-dependent collision time $t_{c}$. The $1 \mathrm{~d}$ profile before collision can be obtained from the literature [26], while after collision it is obtained by solving the $1 \mathrm{~d}$ evolution equation (i.e. $3 \mathrm{~d}$ evolution with spherical symmetry): see Fig. 4 and Ref. [27]. The $1 \mathrm{~d}$ fluid profile generally develops discontinuities (i.e. shocks), which are dealt with using the Kurganov-Tadmor scheme [28] (see Appendix A).

\section{Strategy}

In order to remove the Higgs field from the simulation, we model the system in the following way: first, consider a single bubble with spherical symmetry. Before colliding with surrounding bubbles, the fluid adheres to the conventional self-similar solutions. After the collision, the fluid follows the hydrodynamic equations. After collision it is reasonable to neglect the Higgs field, since it is quickly 
damped to the broken phase [23]. These $1 \mathrm{~d}$ solutions are then embedded into the simulation volume (a similar technique has been used for the scalar field evolution [29]). Every grid point potentially obtains contributions from different bubbles that depend on when the corresponding surface element of the bubble collided with the neighboring bubbles. In this section, we flesh out the details of this approach.

\subsection{Overview}

The GW spectrum is determined from fluid dynamics and the GW waves are stored in the tensor components $h_{i j}$ of the metric

$$
d s^{2}=-d t^{2}+a^{2}\left(\delta_{i j}+h_{i j}\right) d x^{i} d x^{j}
$$

Neglecting cosmic expansion during the transition, the time evolution of $h_{i j}$ for each Fourier component is given by

$$
\ddot{h}_{i j}+k^{2} h_{i j}=\frac{2}{M_{P}^{2}} \Lambda_{i j, k l} T_{k l},
$$

where $M_{P}=1 / \sqrt{8 \pi G}$ is the reduced Planck mass, $\Lambda_{i j, k l}=P_{i k} P_{j l}-P_{i j} P_{k l} / 2$ with $P_{i j}=\delta_{i j}-\hat{k}_{i} \hat{k}_{j}$ is the projection tensor for the transverse-traceless (TT) part, and $T_{i j}$ is the energy-momentum tensor of the system. In our setup the energy-momentum tensor $T_{i j}$ stems from the fluid dynamics during and after transition ${ }^{2}$. Throughout the paper we assume an equation of state for radiation and a perfect fluid

$$
T_{\mu \nu}=w u_{\mu} u_{\nu}+p g_{\mu \nu}
$$

where $w$ and $p$ are enthalpy and pressure, respectively. Notice that only the first term contributes to the GW production. The GW spectrum is then written in terms of the source term using Weinberg's formula

$$
\Omega_{\mathrm{GW}}(q) \equiv \frac{1}{\rho_{\mathrm{tot}}} \frac{d \rho_{\mathrm{GW}}}{d \ln q}=\frac{q^{3}}{4 \pi^{2} \rho_{\mathrm{tot}} M_{P}^{2} V} \int \frac{d \Omega_{k}}{4 \pi}\left[\Lambda_{i j, k l} T_{i j}(q, \vec{k}) T_{k l}^{*}(q, \vec{k})\right]_{q=k} .
$$

Here $\rho_{\text {tot }}$ is the total energy density of the Universe, $q$ and $\vec{k}$ are GW frequency and wavenumber, respectively, with $k \equiv|\vec{k}|$. Also, $V$ is the simulation volume and our convention for the Fourier transform is

$$
T_{i j}(q, \vec{k})=\int d t e^{i q t} \int d^{3} x e^{-i \vec{k} \cdot \vec{x}} T_{i j}(t, \vec{x})
$$

The Fourier transform in the time direction is performed over the simulation time $T$.

In this paper we propose modeling the $3 \mathrm{~d}$ energy-momentum tensor field $T_{i j}$ from $1 \mathrm{~d}$ (more precisely $3 \mathrm{~d}$ with spherical symmetry) hydrodynamic simulations. The main idea is illustrated in Fig. 2. For every bubble we construct the fluid along radially outgoing rays. Depending on whether the corresponding surface element of the bubble already collided with surrounding bubbles or not, we either embed the self-similar solution or the fluid profile obtained from $1 \mathrm{~d}$ hydro simulations.

\footnotetext{
${ }^{2}$ Since the plasma friction makes it difficult for bubbles to run away, the plasma energy dominates over the scalar contribution. The energy contained in the plasma scales with the bubble radius to the third power, while the energy in the scalar field grows with the bubble radius square [30].
} 
Unless the transition is strong ( $\alpha \gtrsim 1$ ), the enthalpy change $\Delta w=w-w_{0}$ and velocity $\vec{v}$ can be treated perturbatively ( $w_{0}$ being the enthalpy deep inside the broken phase, see Fig. 3 ). Since we expect the system to behave perturbatively, the solution of the full simulation is given by the superposition of contributions from different bubbles

$$
\frac{\Delta w}{w_{0}} \simeq \sum_{i: \text { bubbles }} \frac{\Delta w^{(i)}}{w_{0}}, \quad \vec{v} \simeq \sum_{i \text { :bubbles }} \vec{v}^{(i)},
$$

where the superscript $(i)$ denotes the contribution from bubble $i$. Approximating each surface element to be spherically symmetric, the radial profile of the fluid can be obtained from the solution of $1 \mathrm{~d}$ simulations (i.e. $3 \mathrm{~d}$ simulations with spherical symmetry)

$$
\begin{aligned}
\frac{\Delta w^{(i)}}{w_{0}}(t, \vec{x}) & \simeq \frac{\Delta w^{(1 \mathrm{~d})}}{w_{0}}\left(t-t_{n}^{(i)}, t_{c}^{(i)}-t_{n}^{(i)}, r^{(i)}\right), \\
\vec{v}^{(i)}(t, \vec{x}) & \simeq \hat{n}^{(i)} v^{(1 \mathrm{~d})}\left(t-t_{n}^{(i)}, t_{c}^{(i)}-t_{n}^{(i)}, r^{(i)}\right) .
\end{aligned}
$$

Here $\Delta w^{(1 \mathrm{~d})} / w_{0}$ and $v^{(1 \mathrm{~d})}$ are the enthalpy and radial velocity obtained from the $1 \mathrm{~d}$ simulation. The collision time $t_{c}^{(i)}=t_{c}^{(i)}\left(\hat{n}^{(i)}\right)$ depends on the index $i$ for the bubble and the direction $\hat{n}^{(i)}$ of the surface element measured from the bubble nucleation point $\vec{x}_{n}^{(i)}: \hat{n}^{(i)} \equiv \vec{r}^{(i)} /\left|\vec{r}^{(i)}\right|$ with $\vec{r}^{(i)} \equiv \vec{x}-\vec{x}_{n}^{(i)}$. Since the $1 \mathrm{~d}$ solution is common to all the surface elements and all the bubbles, we have to solve the $1 \mathrm{~d}$ evolution only once and then embed the solution into the $3 \mathrm{~d}$ lattice with a proper rescaling with the bubble-dependent nucleation time and direction-dependent collision time. The embedding of the profiles in the grid therefore dominates the execution time of the simulation. In the following subsections, we explain the $1 \mathrm{~d}$ and $3 \mathrm{~d}$ simulations in detail.

\section{$2.21 \mathrm{~d}$ profile}

\subsubsection{Equations, initial conditions, and shocks}

Before bubble collision, the fluid profile is calculated from energy-momentum conservation, $\partial_{\mu} T^{\mu \nu}=$ 0 , together with the energy injection at the wall position [26]. Because of the spherical symmetry of the bubble, the profile becomes self-similar and depends only on $\xi \equiv r /\left(t-t_{n}\right)$. The resulting expansion mode of the bubble has three different types: deflagration, hybrid, and detonation [26].

After bubble collision, the Higgs wall is damped into the broken phase and the fluid is launched into free propagation. This damping occurs on particle physics time scales while the bubble dynamics follows cosmological times scales, and we neglect the Higgs field right after collision. Assuming $d$-dimensional spherical symmetry (i.e. $d=1$ : planar, $d=2$ : cylindrical, $d=3$ : spherical) and a relativistic ideal gas $T_{\mu \nu}=w u_{\mu} u_{\nu}+p g_{\mu \nu}$ with $w=\rho+p$ and $p=\rho / 3$ the evolution equations become (see Refs. [27,31])

$$
\partial_{t} u+\partial_{r} f+g=0
$$

where

$$
u=\left(\begin{array}{l}
u_{1} \\
u_{2}
\end{array}\right)=\left(\begin{array}{c}
w \gamma^{2}-p \\
w \gamma^{2} v
\end{array}\right), \quad f=\left(\begin{array}{c}
w \gamma^{2} v \\
w \gamma^{2} v^{2}+p
\end{array}\right), \quad g=\frac{d-1}{r}\left(\begin{array}{c}
w \gamma^{2} v \\
w \gamma^{2} v^{2}
\end{array}\right)
$$

We solve these equations starting just after the bubble collision with the initial condition set in the following way: we expect that the first crossing of the two profiles do not change their shapes 


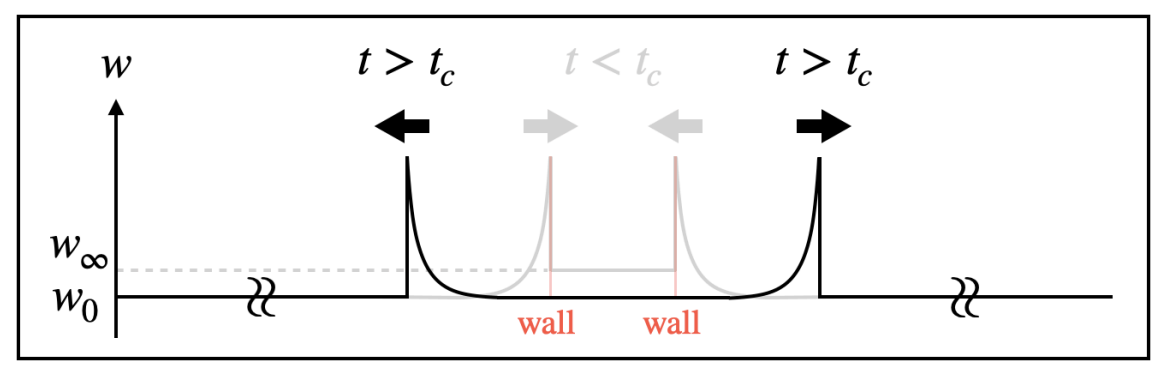

Figure 3: Illustration for the initial condition of the 1d simulation. The figure shows the collision of two fluid profiles. Before collision (gray), the enthalpy takes different values in front $\left(w_{\infty}\right.$, symmetric phase) and behind ( $w_{0}$, broken phase) the profile. Assuming that the first collision does not change the profile significantly, the profile just after collision (black) is given by the same shape as the gray line with the front enthalpy replaced by $w_{0}$. We use the black line as the initial condition for the $1 \mathrm{~d}$ simulation.

significantly as long as the system is in the linear regime (see Fig. 3; this assumption is discussed in more detail in Appendix C). Then the initial condition is given by the self-similar profile of Ref. [26] with the enthalpy in front replaced with that in the broken phase. This is a consequence of the linearity of the system. Figure 3 illustrates how the fluid propagates inside the other bubble (black line) when the profile takes the value in the broken phase.

It is well known that the system (9) can develop discontinuities (shocks). With our initial condition the discontinuity is already established from the beginning at the bubble wall. Solving the hydrodynamic equations numerically requires special attention and we deal with this issue using the method described by Kurganov and Tadmor [28]. The numerical precision of this scheme is discussed in Appendix A. As mentioned before, the execution time is dominated by the embedding into the grid. The $1 \mathrm{~d}$ simulation only takes a few minutes and is not a limiting factor of the accuracy.

\subsubsection{Numerical examples}

In Fig. 4 we show a typical result for the time evolution of the $1 \mathrm{~d}$ profile. The initial condition is taken from the profile in Ref. [26] with $\left(\alpha, \xi_{w}\right)=(0.0046,0.8)$ (corresponding to the maximal fluid velocity at the wall position $v_{\max } \simeq 0.012$ ) with $w$ and $v$ outside replaced with their values inside as explained above. The simulation time is from $t=t_{c}$ (blue line) to $t=7 t_{c}$ (red line).

Here, several comments are in order. First, a sharp discontinuity remains at the front end of the profile even though this simulation is after collision and therefore no bubble wall or energy injection is present in this simulation ${ }^{3}$. As mentioned previously, special care is required for the numerical scheme to retain this feature. We use the Kurganov-Tadmor scheme [28] for this. A more detailed discussion is found in Appendix A. Second, the plot seems to diverge around the origin. This is because of the term $g \propto 1 / r$ in Eq. (9) and depending on the boundary conditions imposed at the origin, the incoming wave will reflect. This is an artifact from the spherical symmetry that would not occur in a more realistic simulation. However, only a very small volume is affected by the assumptions how this singularity is treated and we have checked that this issue does not affect the final GW spectrum in any major ways. Notice that in our model the time dependence is explicit.

\footnotetext{
${ }^{3}$ In the deflagration case, there are two discontinuities: one at the sound shell front and a steeper one at the bubble wall. Evolving the deflagration 1d simulation makes the bubble wall discontinuity eventually catch up with the sound shell front such that it resembles a detonation at late times.
} 

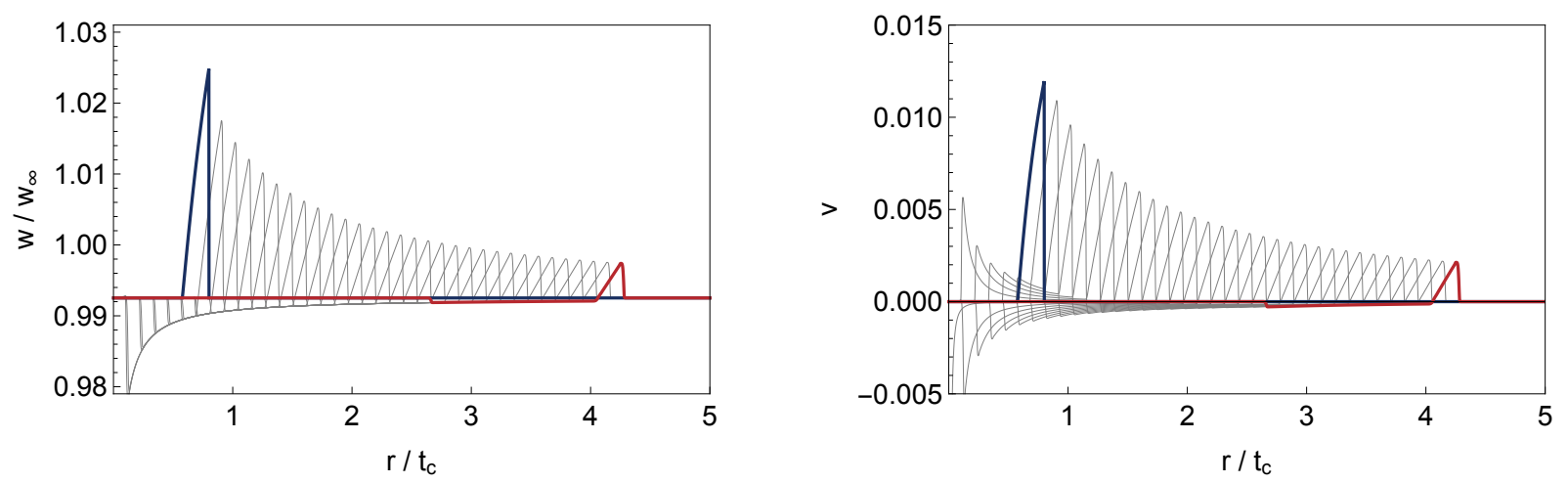

Figure 4: Example of our 1d simulation, showing how the initial condition (blue line) evolves in time. In this plot we used $\alpha=0.0046$ and $\xi_{w}=0.8$ (corresponding to $v_{\max } \simeq 0.012$ ), and evolved the system from $t=t_{c}$ to $7 t_{c}$ (with the nucleation time $t_{n}$ taken to be 0 ).

Once the $1 \mathrm{~d}$ profile is established, the $3 \mathrm{~d}$ embedding can be performed for arbitrary times. Hence, one can perform the simulation with rather large time steps without sacrificing accuracy, unlike real simulations that rely on evolving the equations of motion in time. Finally, when embedding the $1 \mathrm{~d}$ solution in the $3 \mathrm{~d}$ box we sometimes need the $1 \mathrm{~d}$ profile beyond $t=7 t_{c}$ (see also Appendix A for a discussion about the choice of the maximal time of the $1 \mathrm{~d}$ simulation). We extrapolate the $1 \mathrm{~d}$ solution at the last time slice using $v^{(1 \mathrm{~d})} \propto r^{-1}$, i.e.,

$$
\left.v^{(1 \mathrm{~d})}\left(t, t_{c}, r\right)\right|_{t>7 t_{c}}=\frac{\bar{r}}{r} \times\left. v^{(1 \mathrm{~d})}\left(t, t_{c}, \bar{r}\right)\right|_{t=7 t_{c}}
$$

with $\bar{r}=r-c_{s}\left(t-7 t_{c}\right)$ and accordingly for $w-w_{0}$ (we actually extrapolate $\ln \left(w / w_{0}\right)$ in the simulation which is equivalent in leading order).

\section{$2.3 \quad 3 d$ embedding}

Next we discuss how to embed our $1 \mathrm{~d}$ result in the $3 \mathrm{~d}$ simulation. The procedure is illustrated in Fig. 2. We generate nucleation points in a $3 \mathrm{~d}$ box with size $L$. Each bubble has directiondependent first collision time, and we rescale our 1d simulation result for each direction to embed the $1 \mathrm{~d}$ fluid evolution into the $3 \mathrm{~d}$ lattice. From the $3 \mathrm{~d}$ lattice thus constructed, we generate the transverse-traceless energy-momentum tensor field as

$$
\Lambda_{i j, k l} T_{k l}=\Lambda_{i j, k l} \times w \gamma^{2} v_{k} v_{l} .
$$

Our final goal is to calculate the GW spectrum in Eq. (4). However, we can factor out some trivial dependencies from $\Omega_{\mathrm{GW}}$

$$
\Omega_{\mathrm{GW}}=\frac{w^{2} \tau}{4 \pi^{2} \rho_{\mathrm{tot}} M_{P}^{2} \beta} \times Q^{\prime}
$$

where from Eq. (4) the dimensionless quantity $Q^{\prime}$ becomes

$$
Q^{\prime} \equiv \frac{q^{3} \beta}{w^{2} V T} \int \frac{d \Omega_{k}}{4 \pi}\left[\Lambda_{i j, k l} T_{i j}(q, \vec{k}) T_{k l}^{*}(q, \vec{k})\right]_{q=k}
$$


Here $\tau$ denotes the lifetime of the sound waves and the prime means taking the growth rate (with respect to $\beta$-normalized time $\beta t$ ) of the dimensionless quantity $Q .{ }^{4}$ We describe with more details how to obtain the GW spectrum from the velocity embedded grid in Appendix B. Notice that ideally the simulation is large enough that the correlations in the integrals already scale linearly in the integration time and volume, $T \gg 1 / \beta$ and $V \gg 1 / \beta^{3}$. Especially for the soft momentum modes in the IR tail, this is a nontrivial requirement that we checked explicitly. In this limit, $Q$ is a function of $k / \beta$ that depends only on the $1 \mathrm{~d}$ profile that is embedded and the wall velocity. Any dependence on the simulation volume $V$ and simulation time $T$ drops out, while the concrete nucleation history becomes statistically irrelevant (or encoded in the parameter $\beta, \xi_{w}$ and $\alpha$ ).

Notice that the relative factor between $\Omega_{\mathrm{GW}}$ and $Q^{\prime}$ can be recast in terms of the Hubble parameter $H$ as

$$
\frac{\Omega_{\mathrm{GW}}}{Q^{\prime}}=\frac{w^{2} \tau}{4 \pi^{2} \rho_{\mathrm{tot}} M_{P}^{2} \beta} \simeq \frac{4 \rho_{\mathrm{tot}} \tau}{9 \pi^{2} M_{P}^{2} \beta}=\frac{4 H \tau}{3 \pi^{2}} \frac{H}{\beta},
$$

meaning $\Omega_{\mathrm{GW}} \sim Q^{\prime} \times(H / \beta)$ for the sound wave source lasting for the whole Hubble time. Notice that this assumes a weak phase transition and a radiation equation of state, $\Gamma \equiv w / \rho_{\text {tot }} \simeq 4 / 3$.

\section{Main results}

\subsection{Example of the GW spectrum}

We first show an example of our 3d simulation in Fig. 5. The phase transition strength, wall velocity and fluid maximal velocity are $\alpha=0.0046, \xi_{w}=0.8$, and $v_{\max } \simeq 0.012$. The expansion mode is a detonation for this parameter choice. The box size is $V=L^{3}=\left(40 \xi_{w} / \beta\right)^{3}=(32 / \beta)^{3},{ }^{5}$ the grids size is $N^{3}=256^{3}$ with periodic boundary conditions, and the GW spectrum is calculated with the integration range from $t=6 / \beta$ to $22 / \beta$ (see Eq. (5)). This typically results in simulations with about 2500 bubbles. These are the default parameters we use in all simulations if not explicitly stated otherwise.

We notice several features in the spectrum. The spectrum has a single peak at early times $(t \lesssim 12 / \beta)$ while it breaks up into two peaks after that. The peak at early times corresponds to the typical bubble size at the collision time. This is a contribution to the GW spectrum akin to the envelope and bulk flow models that seize after percolation completed. This peak moves to the IR without significant growth in the amplitude, which is qualitatively consistent with Refs. [32,33]. At later time, a second peak develops. This peak is the UV structure peaked at the scale of shell thickness. Ideally, we would like to isolate the late time structure of the simulation that is dominated by the contributions from the sound waves. We come back to this issue in Sec. 3.2.

We define the shell thickness as (see Fig. 6)

$$
\xi_{\text {shell }} \equiv \xi_{\text {front }}-\xi_{\text {rear }}
$$

${ }^{4}$ The definition is

$$
Q \equiv \frac{q^{3} \beta^{2}}{w^{2} V} \int \frac{d \Omega_{k}}{4 \pi}\left[\Lambda_{i j, k l} T_{i j}(q, \vec{k}) T_{k l}^{*}(q, \vec{k})\right]_{q=k}
$$

\footnotetext{
${ }^{5}$ One can use the same nucleation history for different wall velocities. In this case one needs to rescale the box size by the wall velocity to keep the nucleation history to be physical. This is why naturally a factor $\xi_{w}$ comes with the box size.
} 


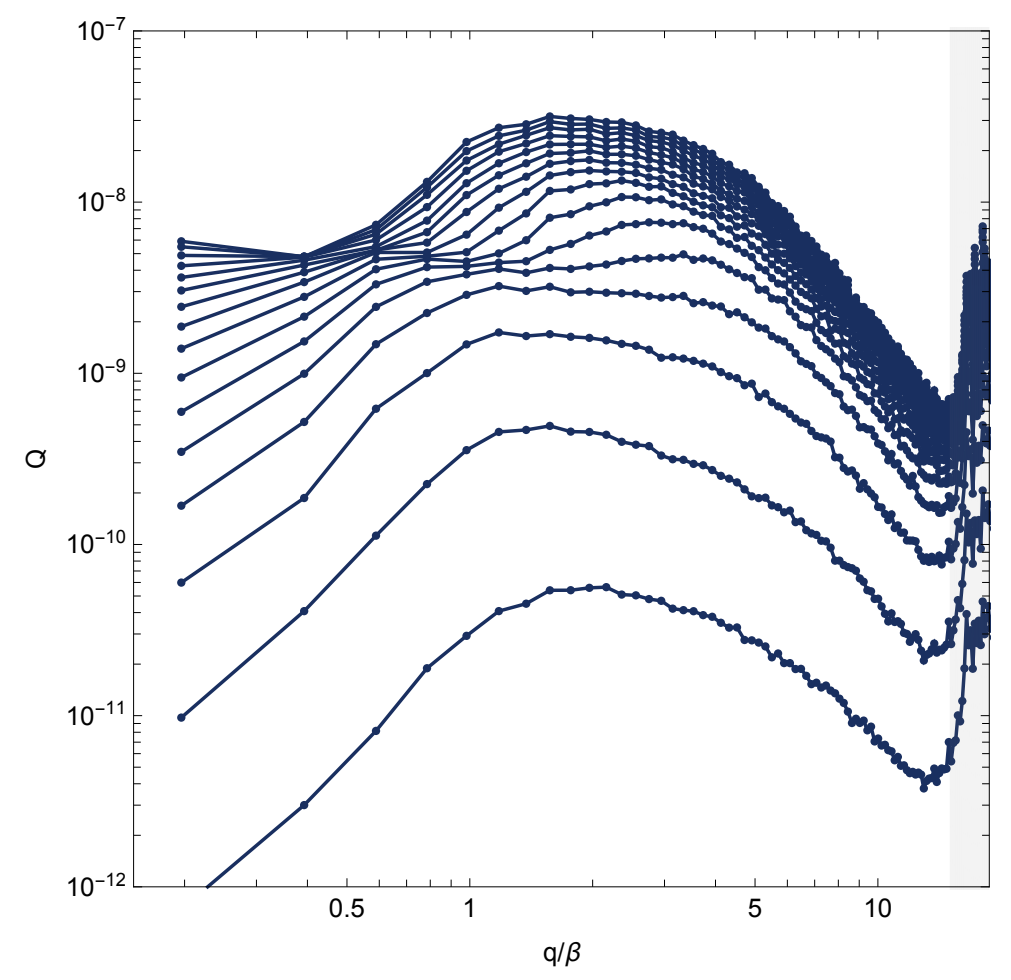

Figure 5: GW spectrum $Q$ for $\alpha=0.0046$ and $\xi_{w}=0.8$ (corresponding to a detonation with $\left.v_{\max } \simeq 0.012\right)$. The box size is $V=L^{3}=\left(40 \xi_{w} / \beta\right)^{3}=(32 / \beta)^{3}$, the number of grids is $N^{3}=$ $256^{3}$, and the integration range is from $t=6 / \beta$ to $22 / \beta$ (the different lines change the upper integration limit in steps of $1 / \beta$ ) with the typical bubble nucleation time being $t \simeq 7 / \beta$. There is a sizable contribution in the IR mostly from the early times (up to percolation) that we suppress by integrating only over rather late times in the following.

where

$$
\xi_{\text {front }}=\left\{\begin{array}{cc}
\xi_{\text {shock }} & (\text { deflagration, hybrid }) \\
\xi_{w} & \text { (detonation) }
\end{array}, \quad \xi_{\text {rear }}=\left\{\begin{array}{cc}
\xi_{w} & \text { (deflagration, hybrid }) \\
c_{s} & \text { (detonation) }
\end{array} .\right.\right.
$$

As we discuss in the next section, the GW spectrum stretches from the (inverse of) typical bubble size at the collision time to the shell thickness.

Following Ref. [25], we consider two types of transitions

$$
\alpha=\left\{\begin{array}{cc}
0.0046 & \text { (weak) } \\
0.05 & \text { (intermediate) }
\end{array}\right.
$$

and take wall velocities from $\xi_{w}=0.32$ to $\xi_{w}=0.8$. Fig. 7 shows examples of the GW spectrum for $\xi_{w}=0.4$ (left columns) and $\xi_{w}=0.52$ (right columns). In the left panels, the two scales in the UV structure are hard to distinguish because the shell thickness $\xi_{\text {shell }} \simeq 0.18$ (for both weak and intermediate phase transitions) is not much different from the bubble size at the collision time (see Sec. 3.2 for a discussion). However, in the right panels the shell thickness is much smaller than the bubble size: $\xi_{\text {shell }} \simeq 0.06$ (weak) and $\xi_{\text {shell }} \simeq 0.07$ (intermediate).

This is why the UV structure stretches to much higher frequencies for the right panels. In Fig. 6 we plot $\xi_{\text {shell }}$ for the weak and intermediate transitions for different wall velocities. Clearly 


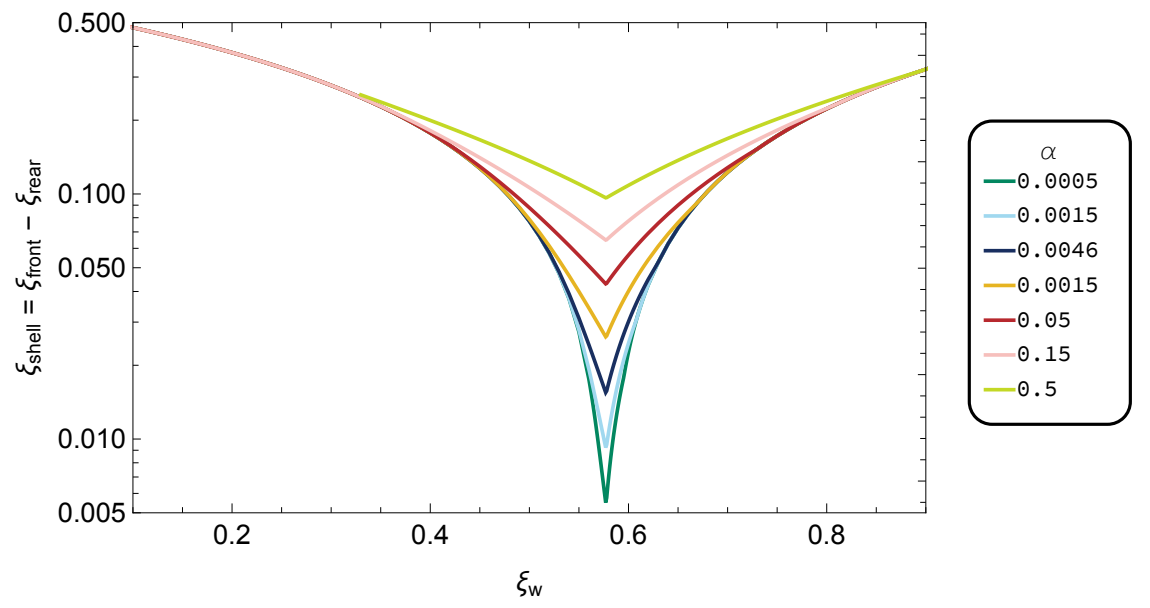

Figure 6: Shell thickness $\xi_{\text {shell }} \equiv \xi_{\text {front }}-\xi_{\text {rear }}$ for different values of $\alpha$ and $\xi_{w}$.

the relation $\xi_{\text {shell }} \simeq\left|\xi_{w}-c_{s}\right|$ holds for weaker transitions while it breaks down (for deflagrations and hybrids) as the transition becomes stronger.

\subsection{Extraction of the linear growth of the spectrum}

Once the typical number of overlapping sound shells at an arbitrary spatial point exceeds unity, the root-mean-square of the fluid velocity remains almost constant in time (until the onset of turbulence) $[18,23-25,34,35]$. The GW spectrum grows linearly in time after that. In other words, the correlation of the energy-momentum tensor at different times dies off if the two times are too dissimilar. Hence, even though the Weinberg formula involves integration over two times, the final result should only be linear in the simulation time. The early dynamics is dominated by the first few bubbles (which leads to a large enhancement in the IR) and we are rather interested in the late time behavior of the simulations. We only integrate over rather late times (from $t=14 / \beta$ to $22 / \beta)$ so that the IR part of the spectrum is reduced. Fig. 8 illustrates this point. For each panel ( $\alpha=0.0046$ or $0.05, \xi_{w}=0.4$ or 0.52$)$ we integrate $T_{i j}$ for a short period $\Delta T=2 / \beta$ (and thus replacing the integration range $T$ in Eq. (5) with $\Delta T$ ). The resulting linear growth $Q^{\prime}$ is almost constant for $t=14 / \beta-22 / \beta$.

Ideally, one would like to express the final GW spectrum in quantities that are easily calculated. The GW spectrum is proportional to the energy-momentum tensor squared and only the enthalpy part of the energy-momentum tensor contributes to the anisotropic stress. Hence one expects a naive scaling $Q^{\prime} \propto\left(\left\langle w \gamma^{2} v^{2}\right\rangle / w_{\infty}\right)^{2}$. However, $\left\langle w \gamma^{2} v^{2}\right\rangle$ can be evaluated at different stages of our simulation leading to different results.

The first representation of $\left\langle w \gamma^{2} v^{2}\right\rangle$ is the kinetic energy contained in the sound shell of the expanding bubble before collision. This is thus calculated from the self-similar profile by

$$
\kappa \alpha=\frac{4}{\xi_{w}^{3} w_{\infty}} \int d \xi w \gamma^{2} v^{2} \xi^{2},
$$

where $w_{\infty}$ is the enthalpy in the symmetric phase. The second and third are average of $w \gamma^{2} v^{2}$ in the $1 \mathrm{~d}$ simulation after collision and the $3 \mathrm{~d}$ value evaluated on the grid of the box, respectively. For $\left\langle w \gamma^{2} v^{2}\right\rangle_{1 \mathrm{~d}}$ we average $w \gamma^{2} v^{2}$ over the spherical volume at the last time slice of the $1 \mathrm{~d}$ simulation $t=7 t_{c}$. For $\left\langle w \gamma^{2} v^{2}\right\rangle_{3 \mathrm{~d}}$ we spatially average $w \gamma^{2} v^{2}$ in the $3 \mathrm{~d}$ box and further average for the period from $t=14 / \beta$ to $22 / \beta$ (which is our integration range for $Q^{\prime}$ ). 

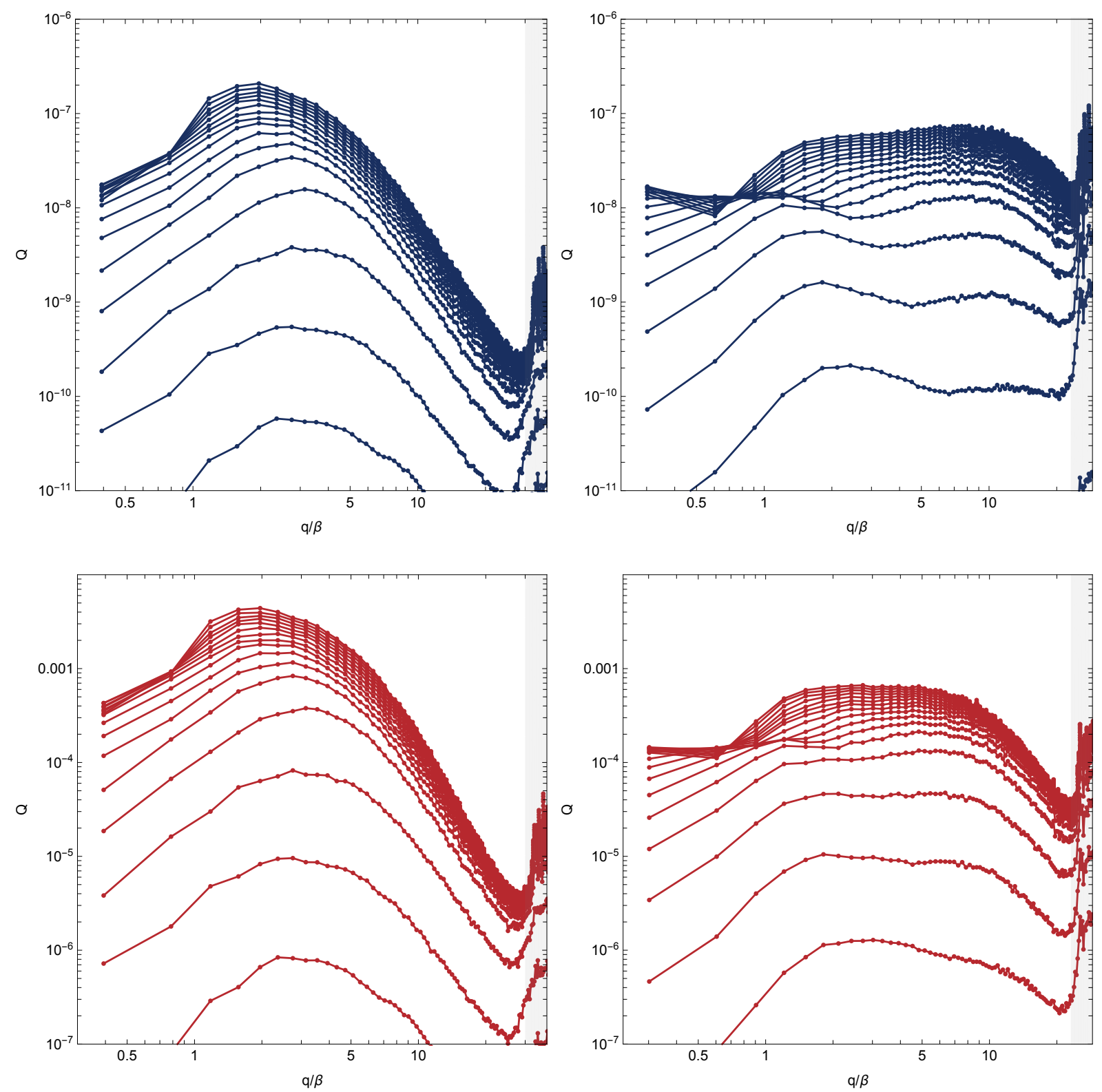

Figure 7: GW spectrum in weak ( $\alpha=0.0046$, top panels) and intermediate $(\alpha=0.05$, bottom panels) transitions. The wall velocity is $\xi_{w}=0.4$ and $\xi_{w}=0.52$ for the left and right panels, respectively. In the right plot a clear separation of the shell thickness and bubble size length scales can be observed. 

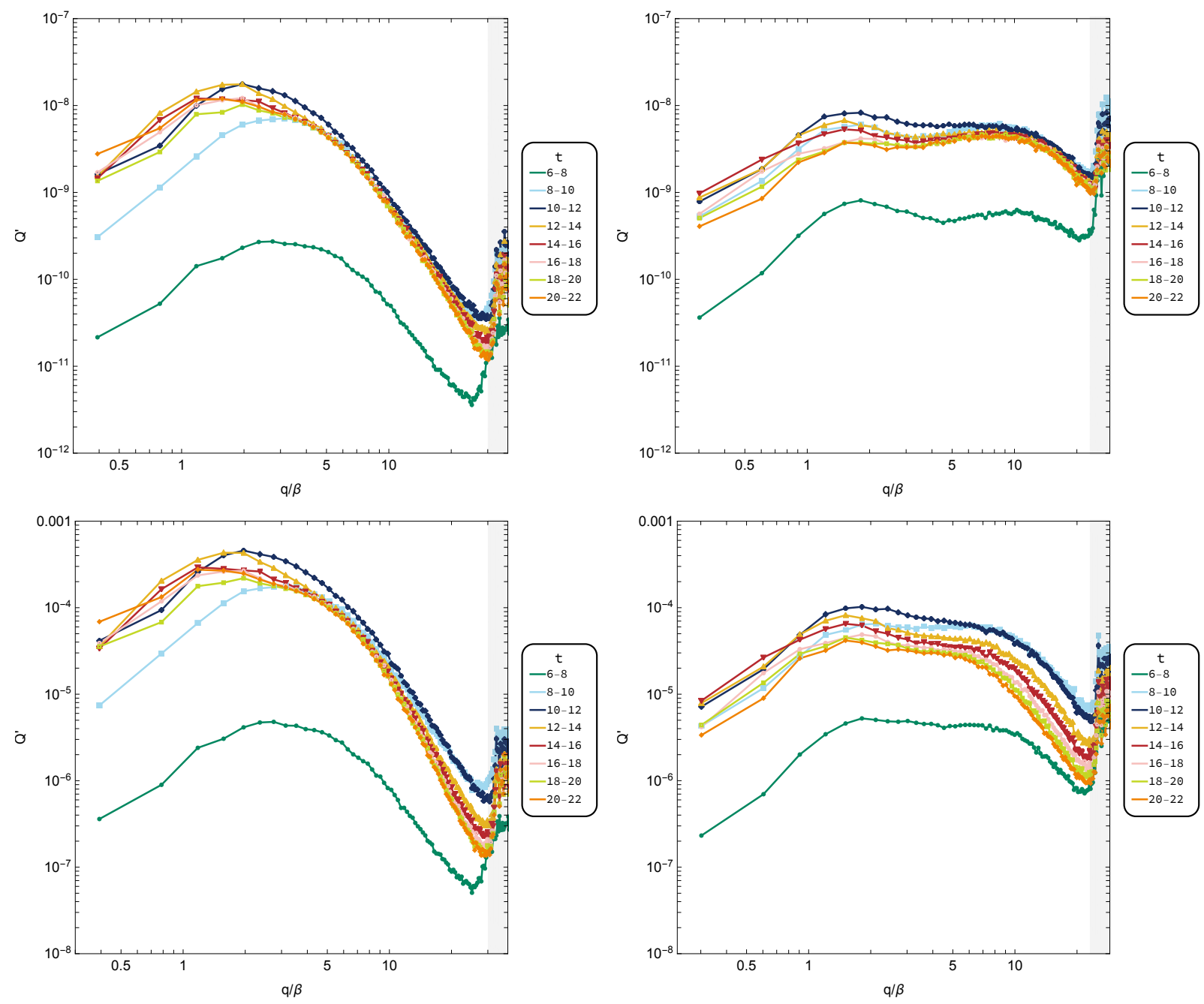

Figure 8: Growth rate of the GW spectrum $Q^{\prime}$ calculated for each short period $\Delta T=2 / \beta$. The four panels correspond to the parameter points in Fig. 7 (top-left: $\alpha=0.0046, \xi_{w}=0.4$, top-right: $\alpha=0.0046, \xi_{w}=0.52$, bottom-left: $\alpha=0.05, \xi_{w}=0.4$, bottom-right: $\left.\alpha=0.05, \xi_{w}=0.52\right)$. In evaluating $Q^{\prime}$ we take the lower and upper limit for the time integration in Eq. (5) to be the range shown in the legend. For $t=14 / \beta-22 / \beta$ the UV part is stable. 
The second question is what observable is most easily related to the model parameters. We studied several possibilities and found that the integrated GW spectrum allows for the most straightforward relation to the model parameters (compared to e.g. the peak of the GW spectrum). We hence use

$$
Q_{\text {int }}^{\prime} \equiv \int d \ln q Q^{\prime}(q)
$$

to study the model dependence. We also found the final GW spectrum scales with the shell thickness, which is due to the fact that the anisotropic stress stems from the overlap of different sound shells rather than the sound shells individually, see Ref. [35].

Figure 9 shows $Q_{\text {int }}^{\prime}$ for various values of $\alpha$ and $\xi_{w}$ and the three parameters quantifying the strength of the phase transition. While $\left\langle w \gamma^{2} v^{2}\right\rangle_{1 \mathrm{~d}}$ and $\left\langle w \gamma^{2} v^{2}\right\rangle_{3 \mathrm{~d}}$ show similar dependence, $\kappa \alpha$ behaves quite differently from the others. As we discuss below and in Appendix D, this comes from the rearrangement of the fluid profile after collision, and this effect is stronger for smaller $\xi_{w}$. As a result, the GW spectrum estimated from $\kappa \alpha$ underestimates the true spectrum by orders of magnitude for small $\xi_{w}$. The strength parameter measured in the $3 \mathrm{~d}$ simulation tracks the GW spectrum excellently, only leading to small variations for wall velocities close to the speed of sound. The strength parameter measured in the $1 \mathrm{~d}$ simulation performs somewhat worse, especially for stronger phase transitions and small wall velocities. This is due to non-linear effects arising in the simulations when many shells overlap in the same location.

Fitting the integrated GW spectrum As shown in the last section, the GW spectrum falls within a factor of $\sim 2-3$ for all the wall velocities for different expansion modes of transitions when normalized by $\left\langle w \gamma^{2} v^{2}\right\rangle_{3 \mathrm{~d}}^{2}$. Hence a reasonable fit to the data is given by

$$
Q_{\text {int }}^{\prime} \simeq 9 \times \xi_{\text {shell }} \times\left(\left\langle w \gamma^{2} v^{2}\right\rangle_{3 \mathrm{~d}} / w_{\infty}\right)^{2} .
$$

In this expression the normalization coefficient $\left\langle w \gamma^{2} v^{2}\right\rangle_{3 \mathrm{~d}}$ is the $3 \mathrm{~d}$ strength measured in the simulation.

More readily obtained is the $1 \mathrm{~d}$ strength parameter, since this only requires a $1 \mathrm{~d}$ simulation which is not as demanding. As seen from Fig. 9, $\left\langle w \gamma^{2} v^{2}\right\rangle_{1 \mathrm{~d}}$ also gives a reasonable normalization of the spectrum in the weak transition regime. The data points for $\alpha=0.05$ start to deviate from others because of nonlinear effects: while $\Delta w^{(i)} / w_{0}$ and $\vec{v}^{(i)}$ from each bubble is well within the perturbative regime, the velocity sum can be $\mathcal{O}(0.1)$ on some grid points. This makes $w \gamma^{2} v^{2}$ on the $3 \mathrm{~d}$ grids larger than $w \gamma^{2} v^{2}$ estimated from a single bubble. From our data we obtain

$$
Q_{\text {int }}^{\prime} \simeq 12 \times \xi_{\text {shell }} \times\left(\left\langle w \gamma^{2} v^{2}\right\rangle_{1 \mathrm{~d}} / w_{\infty}\right)^{2} .
$$

This estimate is conservative and underestimates the GW signal for the case of strong phase transitions or wall velocities close to the speed of sound.

Finally, normalization by $\kappa \alpha$ fails badly as seen from Fig. 9 . This is because $\kappa \alpha$, the fluid kinetic energy estimated from an expanding bubble (before collision), is not a good measure for the kinetic energy after bubbles collide. Main reason is that at late times, the sound shells resemble detonations with a shock front and a rarefaction wave at late times. Hence the fluid profile has to rearrange significantly, especially for small $\xi_{w}$. As seen from the top panel of Fig. 16 (see Appendix D), the fluid kinetic energy changes by a factor of $\sim 4-5$ for $\xi_{w}=0.32$ just after collision. This is by no means in contradiction with energy-momentum conservation, since excess pressure (that dominates the energy-momentum tensor in the linear regime) can be readily converted into kinetic 

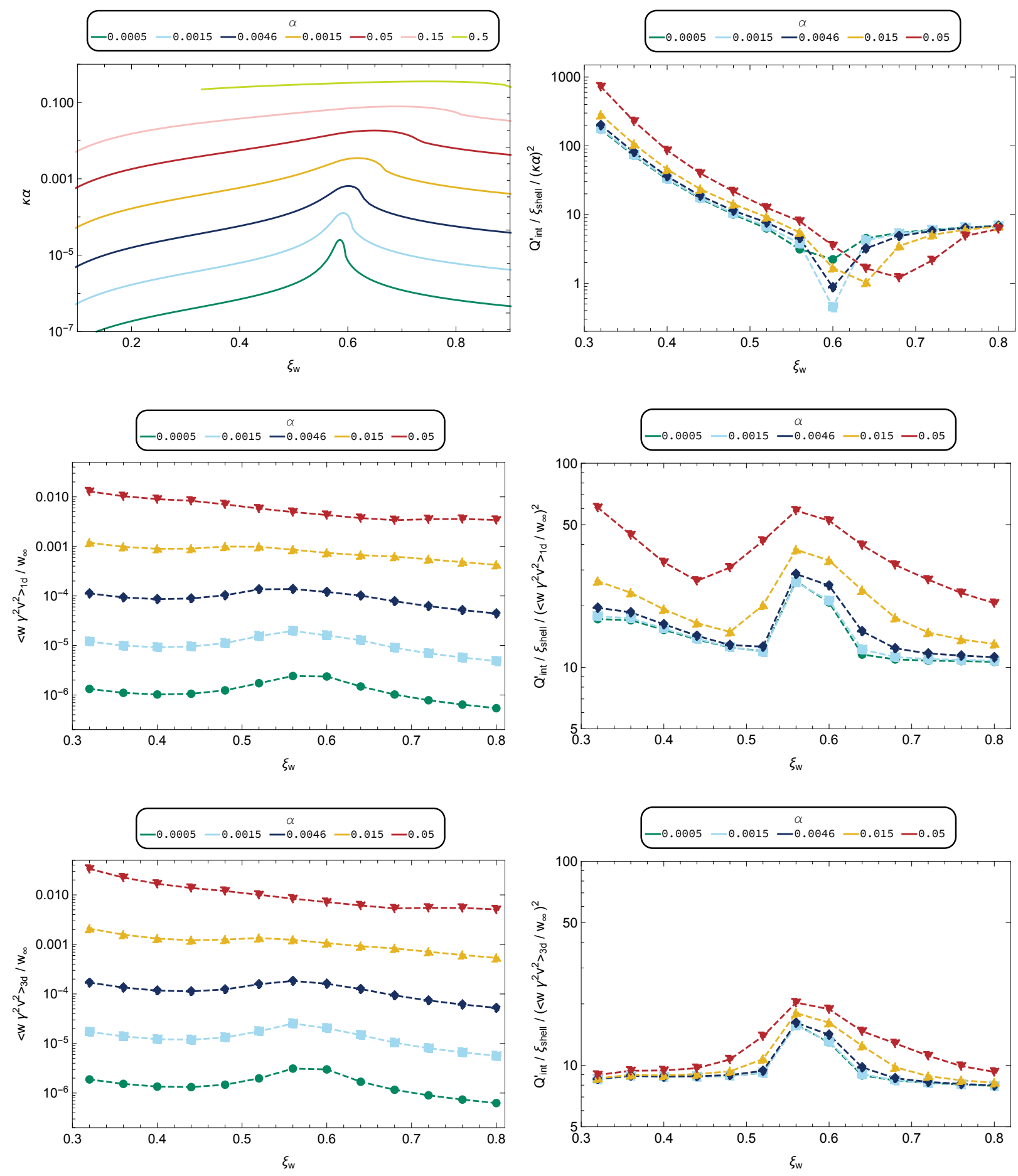

Figure 9: The left plots show the possible normalization factors $\kappa \alpha,\left\langle w \gamma^{2} v^{2}\right\rangle_{1 \mathrm{~d}} / w_{\infty}$, and $\left\langle w \gamma^{2} v^{2}\right\rangle_{3 \mathrm{~d}} / w_{\infty}$ for different values of $\alpha$ and $\xi_{w}$. The right plot shows the integrated growth factor $Q_{\text {int }}^{\prime}$ in relations to the normalization factors. 


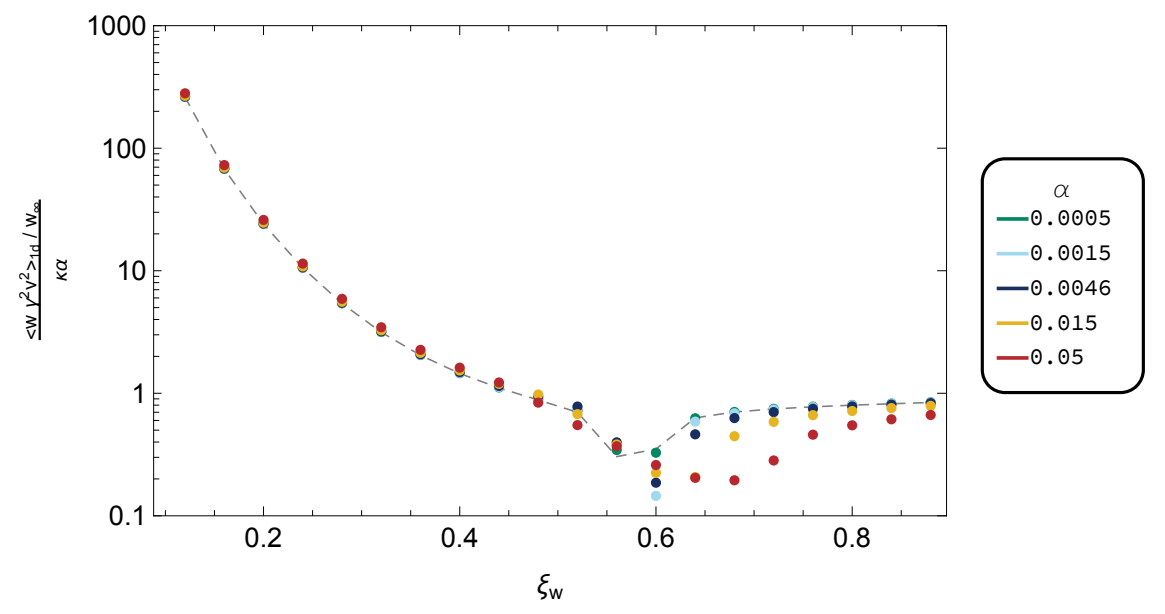

Figure 10: Ratio between $\kappa \alpha$ and $\left\langle w \gamma^{2} v^{2}\right\rangle_{1 \mathrm{~d}} / w_{\infty}$ for different values of $\alpha$ and $\xi_{w}$. The gray dashed line is drawn from data for $\alpha=0.0001$.

energy when the fluid shell splits into a right- and left-moving wave (we checked energy-momentum conservation explicitly in our $1 \mathrm{~d}$ simulations). As a consequence, using $Q_{\mathrm{int}}^{\prime} \sim($ const. $) \times(\kappa \alpha)^{2} \xi_{\text {shell }}$ significantly underestimates the GW spectrum for small $\xi_{w}$ in our simulations. Of course, to what extent this effect persists in the non-linear regime is debatable, since we assume linearity when the fluid is embedded into the grid.

In Fig. 10 we plot the relation between $\kappa \alpha$ and $\left\langle w \gamma^{2} v^{2}\right\rangle_{1 \mathrm{~d}}$. The colored data points are for $\alpha=0.0005,0.015, \cdots, 0.05$ while the gray line is drawn from $\alpha=0.0001$. Interestingly, the relation seems universal and not to depend strongly on $\alpha$ for small wall velocities. Hence, this figure can be readily used to translate $\kappa \alpha$ into $\left\langle w \gamma^{2} v^{2}\right\rangle_{1 \mathrm{~d}}$. Special care has to be used for wall velocities close to the speed of sound, since the GW spectrum from the simulation does not show a significant increase for hybrids, unlike $\kappa \alpha$.

Fitting the spectral shape A detailed comparison with experimental sensitivity curves also requires the knowledge of the peak frequency (or frequencies) of the spectrum and to certain extent the asymptotic behavior. We fitted all spectra to a double-broken power law

$$
\begin{aligned}
\frac{Q^{\prime}}{\xi_{\text {shell }} \times\left(\left\langle w \gamma^{2} v^{2}\right\rangle_{3 \mathrm{~d}} / w_{\infty}\right)^{2}} & \propto \frac{1}{\left(q / q_{l}\right)^{-n_{l}}+\left(q / q_{l}\right)^{-n_{m}}+\left(q_{h} / q_{l}\right)^{-n_{m}}\left(q / q_{h}\right)^{-n_{h}}} \\
& \simeq\left\{\begin{array}{cc}
\left(q / q_{l}\right)^{n_{l}} & \left(q \ll q_{l}\right) \\
\left(q / q_{l}\right)^{n_{m}} & \left(q_{l} \ll q \ll q_{h}\right) . \\
\left(q_{h} / q_{l}\right)^{n_{m}}\left(q / q_{h}\right)^{n_{h}} & \left(q_{h} \ll q\right)
\end{array}\right.
\end{aligned}
$$

Note that the template is proportional to $q^{n_{l}}, q^{n_{m}}$, and $q^{n_{h}}$ for the three frequency ranges, respectively. Fig. 11 shows the fitting result. The amplitude can be determined via the fit of the integrated GW spectrum, c.f. Eq. (21).

One sees that the bending at the low frequency is almost constant in $\xi_{w}$ while the one at high frequency correlates with the shell thickness. This is somewhat unexpected, since one would expect that the (physical) bubble size scales with the wall velocity. However, the fluid profile for (subsonic) deflagrations actually ends with the shock front that propagates at least as fast as the speed of 

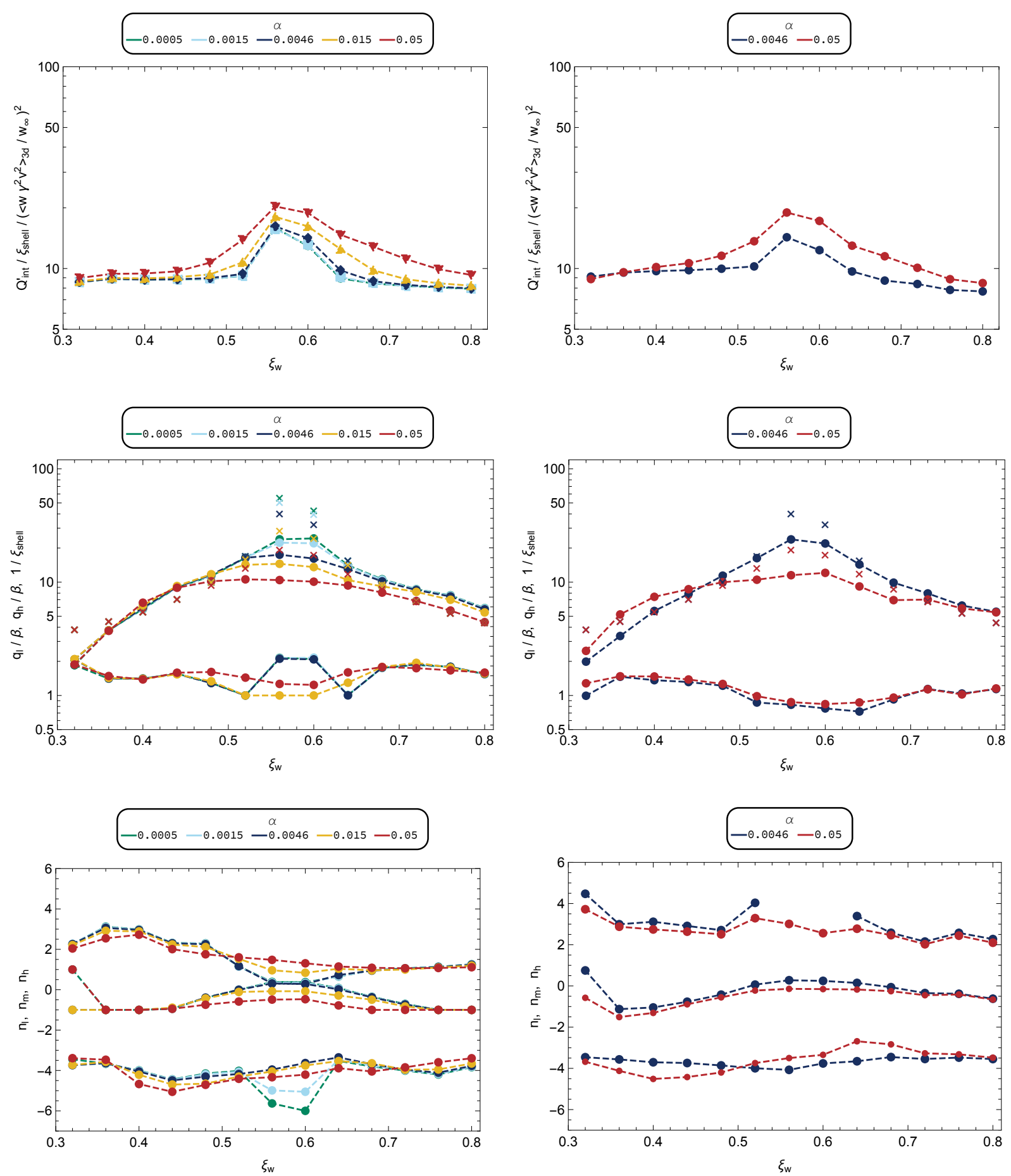

Figure 11: The plots show the fitting parameters in Eq. (23) of the GW spectrum for $N=256$ and $L=40 \xi_{w} / \beta$ (left) and $N=512$ and $L=80 \xi_{w} / \beta$ (right). Missing points indicate that the corresponding parameters could not be measured reasonably due to different issues (dominance by GWs from collisions or a lack of resolution). We can see that some features for thin sound shells are better resolved in the high-resolution simulations. The crosses indicate the inverse shell thickness that should be compared to the position of the second peak in the spectrum. 
sound. Also for detonations no decrease in the momentum scale coming from the bubble size can be observed. Even though we simulate phase transition with wall velocities up to $\xi_{w}=0.8$, there is no clear trend in the data.

We also performed simulations with a bigger box in order to investigate the box size effect (especially on the IR exponent). The right panels of Fig. 11 use $L=80 \xi_{w} / \beta$ and $N=512$, while the left plots are smaller simulations with $L=40 \xi_{w} / \beta$ and $N=256$. For most parts, this allows to measure the IR behavior with better accuracy. Our data is consistent with

$$
q_{l} \simeq 1, \quad q_{h} \simeq 1 / \xi_{\text {shell }},
$$

and

$$
n_{l} \in[2,4], \quad n_{m} \in[-1,0], \quad n_{h} \in[-4,-3],
$$

This combines the steep rise of the envelope approximation with the fast fall-off from the bulk flow model.

We finally summarize how to use our result. For given $\alpha$ and $\xi_{w}$ one can calculate the kinetic energy fraction before collision $\kappa \alpha$ and the shell thickness $\xi_{\text {shell }}$ from the fluid profile of an expanding bubble using Refs. $[22,26,36]$. It can be converted to the 1d kinetic energy long after collision $\left\langle w \gamma^{2} v^{2}\right\rangle_{1 \mathrm{~d}} / w_{\infty}$ using Fig. 10. Then the linear growth of the GW spectrum $Q^{\prime}$ is calculated from Eq. (24) with the overall normalization given by Eq. (22). The parameters characterizing the spectral shape can be read off from Fig. 11 (left: small box simulation, right: big box simulation) or Eqs. (25) and (26). Finally $Q^{\prime}$ can be converted to the GW spectrum $\Omega_{\mathrm{GW}}$ using Eq. (13).

\section{Discussion and conclusions}

In this paper we proposed a novel way to calculate the sound wave contribution to the GW spectrum in first-order phase transitions. The main idea is illustrated in Fig. 2: assuming linearized fluid equations of motion after the transition, both the enthalpy and velocity field are well described by the superposition of contributions from different bubbles. The contribution from each bubble can be calculated from $1 \mathrm{~d}$ simulation (i.e. $3 \mathrm{~d}$ with spherical symmetry) with a proper rescaling of the collision time depending on the direction of the surface element of each bubble. One of the advantages of our method is that we can incorporate the shock front (discontinuities in fluid profile) relatively easily. Hence, our scheme only contains the scalar field as a boundary condition in the $1 \mathrm{~d}$ simulation. The dynamical range of the simulation has to resolve only the bubble size and the sound shell thickness but not the bubble wall thickness. This allows for economic simulations and more extensive parameter scans.

In our setup, it is possible to separate the mean bubble separation from the simulation volume (we always spawn more than 2500 bubbles in our simulation) and to separate the shell thickness from the grid spacing more easily. For lattice simulations including the scalar field this is prohibitively expensive in the regime of thin sound shells when the wall velocity is close to the speed of sound.

We discussed the dependence of the resulting GW spectrum on the fluid kinetic energy and on the shell thickness, and successfully scaled out the proportionality factor. We provide expressions for the contributions of the GW spectrum for sound waves from fitting our data in the last section. Overall, the most robust outcome of our simulations is that the best observable to benchmark is the integrated power spectrum. When normalized to the kinetic energy at late times in the simulation, $\left\langle w \gamma^{2} v^{2}\right\rangle_{3 \mathrm{~d}}$, the results become rather independent from the wall velocity and strength of the phase transition, see Fig. 9. 
In comparison with former lattice results of the fluid interacting with a scalar field [25] we find the same qualitative features. The mean bubble separation and the sound shell thickness leave both traces in the power spectrum in the form of a double-broken power law. However, quantitatively we find some differences, see Fig. 11. Overall, our GW signal is somewhat larger (by about a factor $\sim 2$ for $\alpha=0.0046$ and $\xi_{w}=0.8$ ). We also find a rather flat spectrum (a plateau) between the scale of the bubble separation and the shell thickness, while the lattice results and the sound shell model [34] seem to support a linear increase, $\Omega_{\mathrm{GW}} \sim k$. Some of these differences can be attributed to the fact that we nucleate bubbles according to an exponential increase in probability compared to simultaneous nucleations. Besides, the bubble count in our simulations is higher, which tends to reduce finite volume effects and slightly increases the result.

Moreover, our results indicate that the spectrum is rather independent of the wall velocity when normalized to the measured kinetic energy $\left\langle w \gamma^{2} v^{2}\right\rangle_{3 \mathrm{~d}}$, just as the lattice simulations indicate. However, we observe an enhancement of the kinetic energy in our simulation, $\left\langle w \gamma^{2} v^{2}\right\rangle_{3 \mathrm{~d}}$, compared to the kinetic energy inferred from the self-similar fluid profiles before collision, $\kappa \alpha$, for small wall velocities, see Fig. 10. Hence, we predict a significantly larger GW signal for small wall velocities than naively estimated from $\kappa \alpha$. We only find a modest enhancement in the GW signal for wall velocities close to the speed of sound, unlike the large enhancement seen in $\kappa \alpha$. While lattice simulations in this regime are very hard, our results predict a smaller signal than previously believed in this case.

\section{Acknowledgment}

The work of RJ was supported by Grants-in-Aid for JSPS Overseas Research Fellow (No. 201960698). This work is supported by the Deutsche Forschungsgemeinschaft under Germany's Excellence Strategy - EXC 2121 „Quantum Universe“ - 390833306.

\section{A Numerical Evolution of the 1d profile}

\section{A.1 Equations}

We solve the hydrodynamic equations $\partial_{\mu} T^{\mu \nu}=0$ and assume $d$-dimensional spherical symmetry (e.g. $d=1$ is planar, $d=2$ is cylindrical, and $d=3$ is spherical). We also assume a relativistic ideal gas $T_{\mu \nu}=w u_{\mu} u_{\nu}+p g_{\mu \nu}$ with $w=\rho+p$ and $p=\rho / 3$. This leads to the evolution equations already stated in Eq. (9)

$$
\partial_{t} u+\partial_{r} f+g=0
$$

where

$$
u=\left(\begin{array}{l}
u_{1} \\
u_{2}
\end{array}\right)=\left(\begin{array}{c}
w \gamma^{2}-p \\
w \gamma^{2} v
\end{array}\right), \quad f=\left(\begin{array}{c}
w \gamma^{2} v \\
w \gamma^{2} v^{2}+p
\end{array}\right), \quad g=\frac{d-1}{r}\left(\begin{array}{c}
w \gamma^{2} v \\
w \gamma^{2} v^{2}
\end{array}\right) .
$$

In terms of $\rho$ and $v$, we have

$$
\partial_{t}\left(\begin{array}{l}
\rho \\
v
\end{array}\right)+A \partial_{r}\left(\begin{array}{l}
\rho \\
v
\end{array}\right)+h=0
$$


where $A$ and $h$ are

$$
A=\frac{1}{1-c_{s}^{2} v^{2}}\left(\begin{array}{cc}
\left(1-c_{s}^{2}\right) v & \rho+p \\
\frac{c_{s}^{2}\left(1-v^{2}\right)^{2}}{\rho+p} & \left(1-c_{s}^{2}\right) v
\end{array}\right), \quad h=\frac{d-1}{r}\left(\begin{array}{c}
\frac{(\rho+p) v}{1-c_{s}^{2} v^{2}} \\
-\frac{c_{s}^{2} v^{2}\left(1-v^{2}\right)}{1-c_{s}^{2} v^{2}}
\end{array}\right) .
$$

For $p=\rho / 3$, we can write $f$ and $g$ in terms of $u$ as

$$
u=\left(\begin{array}{l}
u_{1} \\
u_{2}
\end{array}\right), \quad f=\left(\begin{array}{c}
u_{2} \\
\frac{5}{3} u_{1}-\frac{2}{3} \sqrt{4 u_{1}^{2}-3 u_{2}^{2}},
\end{array}\right), g=\frac{d-1}{r}\left(\begin{array}{c}
u_{2} \\
2 u_{1}-\sqrt{4 u_{1}^{2}-3 u_{2}^{2}}
\end{array}\right) .
$$

Here we used

$$
u_{1}=\frac{1+v^{2} / 3}{1-v^{2}} \rho, \quad u_{2}=\frac{4 v / 3}{1-v^{2}} \rho, \quad v=2\left(\frac{u_{1}}{u_{2}}\right)-\sqrt{4\left(\frac{u_{1}}{u_{2}}\right)^{2}-3}=\frac{3 u_{2}}{2 u_{1}+\sqrt{4 u_{1}^{2}-3 u_{2}^{2}}} .
$$

\section{A.2 Numerical schemes}

In this appendix, we explain the numerical scheme adopted to evolve the $1 \mathrm{~d}$ fluid dynamics, described by the equation system above. This kind of convective-diffusive systems admits a class of finite-difference solution schemes that are independent of the eigenvalues of the function $f$, called central schemes. One of the simplest and widely-used schemes is the so-called Lax-Friedrichs (LF) scheme $[37,38]$

$$
u_{j}^{n+1}=\frac{u_{j+1}^{n}+u_{j-1}^{n}}{2}-\frac{\lambda}{2}\left[f\left(u_{j+1}^{n}\right)-f\left(u_{j-1}^{n}\right)\right]-g_{j}^{n} \Delta t,
$$

with $\lambda=\Delta t / \Delta x^{6}$. The upper and bottom indices denote the time and space lattice index, respectively. But the LF scheme, as will be shown below, has a large numerical viscosity, that demands a huge resolution to solve the shockwave fronts. The Kurganov-Tadmor (KT) discretization scheme, introduced in [28], reduces the numerical viscosity. The evolution of a lattice site is given by

$$
u_{j}^{n+1}=u_{j}^{n}-\lambda\left[H_{j+1 / 2}\left(t^{n}\right)-H_{j-1 / 2}\left(t^{n}\right)\right]-g_{j}^{n} \Delta t,
$$

where $H$ is defined between two lattice points as

$$
H_{j+1 / 2}(t)=\frac{f\left(u_{j+1 / 2}^{+}(t)\right)+f\left(u_{j+1 / 2}^{-}(t)\right)}{2}-\frac{a_{j+1 / 2}(t)}{2}\left[u_{j+1 / 2}^{+}(t)-u_{j+1 / 2}^{-}(t)\right] .
$$

The value of $u_{j+1 / 2}^{+}$is calculated through a decrement of $u_{j+1}$ while $u_{j+1 / 2}^{-}$is calculated as an increment of $u_{j}$

$$
\begin{aligned}
u_{j+1 / 2}^{+}(t) & =u_{j+1}(t)-\frac{\Delta x}{2}\left(u_{x}\right)_{j+1}(t) \\
u_{j+1 / 2}^{-}(t) & =u_{j}(t)+\frac{\Delta x}{2}\left(u_{x}\right)_{j}(t) .
\end{aligned}
$$

\footnotetext{
${ }^{6}$ It is important to guarantee that $\lambda \lesssim 0.5$, such that $\Delta t$ is smaller than $\Delta x$ and one can solve the shockwave elements.
} 
The derivative of $u$ at the site $j,\left(u_{x}\right)_{j}$ is calculated through

$$
\left(u_{x}\right)_{j}=\operatorname{minmod}\left(\theta \frac{u_{j}-u_{j-1}}{\Delta x}, \frac{u_{j+1}-u_{j-1}}{2 \Delta x}, \theta \frac{u_{j+1}-u_{j}}{\Delta x}\right)
$$

where minmod is equal to the minimum of the elements if all of them are positive, the maximum of the elements if all of them are negative, and zero otherwise. The value of $a_{j+1 / 2}(t)$ is defined as the maximum local speed of the fluid element in that intermediate cell, thus one needs to calculate the maximum value of the fluid velocity using both Eqs. (36) and (37) and boost it by the sound speed:

$$
a_{j+1 / 2}(t)=\max \left(\left|\mu\left(v_{j+1 / 2}^{+}, c_{s}\right)\right|,\left|\mu\left(v_{j+1 / 2}^{+},-c_{s}\right)\right|,\left|\mu\left(v_{j+1 / 2}^{-}, c_{s}\right)\right|,\left|\mu\left(v_{j+1 / 2}^{-},-c_{s}\right)\right|\right),
$$

with $\mu(a, b)=(a-b) /(1-a b)$ designating the Lorentz-transformed fluid velocity.

The minmod derivative works as a flux limiter reducing spurious oscillations, which is recurrent in numerical schemes that aim to solve shocks and discontinuities. The free parameter $\theta$ characterizes the strength of the limiter. A small $\theta$ corresponds to smaller derivatives (in absolute value) and increases the numerical drag. Increasing $\theta$ makes the flux limiter weaker and introduces spurious numerical oscillations. In Figure 12 we display the velocity and enthalpy profile for the $1 \mathrm{~d}$ simulation for a detonation with $\xi_{w}=0.8$ a few times steps after the collision. We can see that the LF scheme has a huge numerical viscosity and the shockwave quickly develops into a smoother wave-packet. Also the thickness of the line corresponds to unstable numerical oscillations. We also show different simulations using the KT scheme for different values of $\theta$ inside the range $[1,2]$. For the same lattice parameters $\left(\Delta x=10^{-3}\right.$ and $\left.\Delta t=10^{-4}\right)$, the KT scheme is much more stable which helps to maintain the shockwaves for longer in the 1d simulation, without any kind of spurious smoothing. In the same panels, in dotted, we display the results of a LF scheme with space and time grid 10 times smaller (high res.). Despite the higher resolution the fluid still does not develop a shock front. Also notice that there is no big difference among the KT lines in the chosen $\theta$ range, such that our result agrees with Ref. [28].
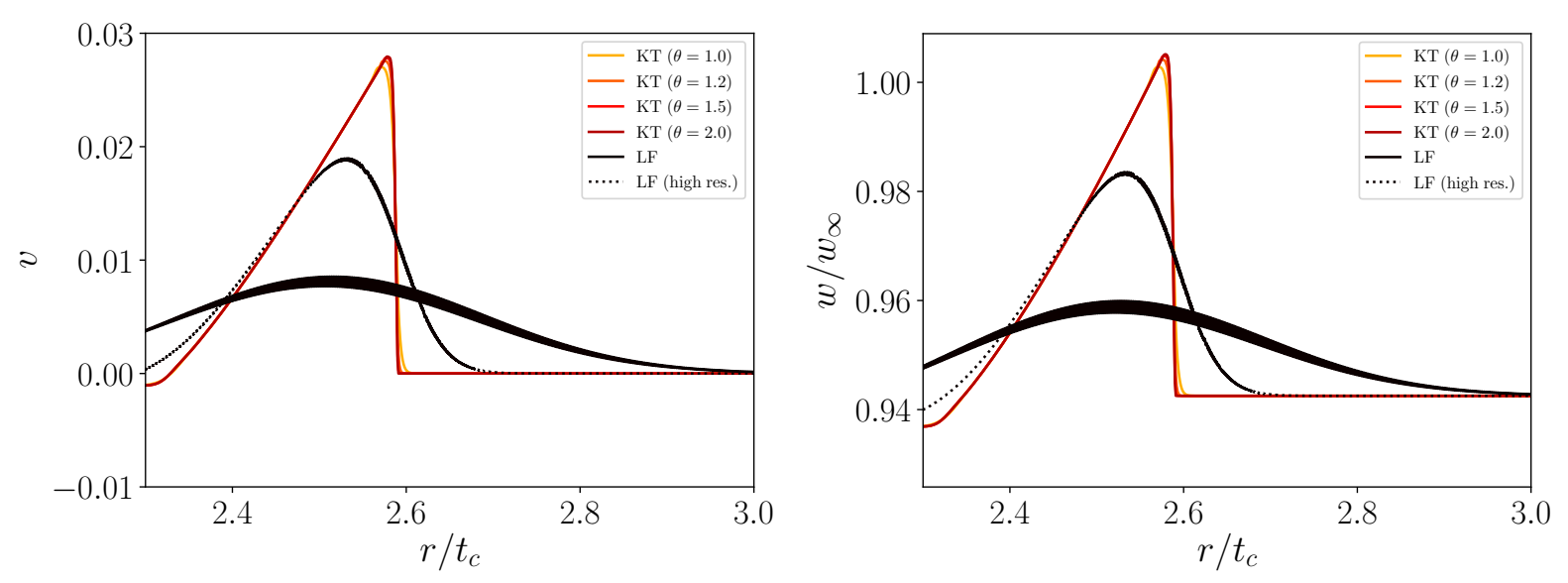

Figure 12: Velocity and enthalpy profiles a few time steps after collision. We used a initial detonation profile with $\xi_{w}=0.8$. We compare the Kurganov-Tadmor (KT) scheme with different values of $\theta$ to the Lax-Friedrichs (LF) scheme. Notice that the LF scheme has some spurious oscillations (shown by the line thickness) and a huge numerical viscosity. For the KT scheme, all values of $\theta$ in the range shown have similar results. Decreasing $\theta$ leads to a larger viscosity and increasing it enhances numerical oscillations. 
The Kurganov-Tadmor method guarantees stability with respect to the spatial discretization. In order to guarantee the stability with respect to time discretization, one can use a Runge-Kutta method (of order $l$ )

$$
\begin{aligned}
u_{j}^{(1)} & =u^{n}+\Delta t C\left[u^{n}\right], \\
u_{j}^{(l+1)} & =\eta_{l} u^{n}+\left(1-\eta_{l}\right)\left(u^{(l)}+\Delta t C\left[u^{(l)}\right]\right), \\
u^{n+1} & =u^{(l)},
\end{aligned}
$$

with the conventional constants $\eta_{l} . C$ is calculated as

$$
C\left[u^{\bullet}\right]=-\left[\frac{H_{j+1 / 2}\left(u^{\bullet}\right)-H_{j-1 / 2}\left(u^{\bullet}\right)}{\Delta x}\right]
$$

In this work, we use the Runge-Kutta method of third order $(l=3)$, i.e. $\eta_{1}=3 / 4$ and $\eta_{2}=1 / 3$, which we found guarantees fast convergence in $\Delta t$.

Finally, we comment on the typical values of $\Delta t$ and $\Delta x$ used for the $1 \mathrm{~d}$ simulations. The typical time scale $t$ and spatial scale $r$ in the $1 \mathrm{~d}$ simulation are given respectively by

$$
\frac{t-t_{n}}{t_{c}-t_{n}}, \frac{r}{t_{c}-t_{n}},
$$

with $t$ the simulation time (typically $\sim 10 / \beta$ ) and $r$ the radial distance between the nucleating points and the grid cell (typically the simulation box size). The value $t_{c}$ is the collision time of the grid point and $t_{n}$ is the bubble nucleation time. Notice that since the surface element of the bubble might collide just after nucleating, $t_{c}-t_{n}$, can be arbitrarily small and consequently the $1 \mathrm{~d}$ simulation time can be arbitrarily large. To overcome this issue, we extrapolate the profile after a maximum $1 \mathrm{~d}$ simulation time $t_{\max }$ using

$$
f(r, t)=f\left(\bar{r}, t_{\max }\right) \times \frac{\bar{r}}{r} \quad \text { with } \quad \bar{r}=r-c_{s}\left(t-t_{\max }\right) .
$$

The final profile (and the GW outcome) has shown to be stable using $t_{\max }$ in the interval $[3,6]$ after collision time (in $1 / \beta$ units), see Fig. $13 .{ }^{7}$ Increasing $t_{\max }$ too much implies not only running the $1 \mathrm{~d}$ simulation for longer times but also reducing $\Delta t$ (and $\Delta x$ ) to overcome numerical friction. Having those scales in mind, the typical lattice size for which the $1 \mathrm{~d}$ simulation is stable is $\Delta x=\mathcal{O}\left(10^{-3}\right)$ and $\Delta t=\mathcal{O}\left(10^{-4}\right)$ using KT scheme and Runge-Kutta of third order.

\section{B From the 3d velocity grid to the GW spectra}

In this appendix, we describe the numerical procedure used in this work to calculate the GW spectra from the velocity grid. Whereas in Section 2 we described the overall set up and how to obtain the velocity grid, here the details of the scheme used to calculate $Q$ from the vector field $v^{i}(\vec{x})$ defined in the lattice site $\vec{x}=\left(x_{i}, y_{j}, z_{k}\right)$, where the indexes $x_{i}, y_{i}$ and $z_{i}$ run from 0 to $N-1$.

The first step is to construct the fluid stress-energy tensor in real space through

$$
T^{i j}(\vec{x})=w(\vec{x}) v^{i}(\vec{x}) v^{j}(\vec{x}) .
$$

\footnotetext{
${ }^{7}$ As stated in the main text, in this work we extrapolate after $t=7 t_{c}$, which corresponds to $t_{\max }=6$.
} 

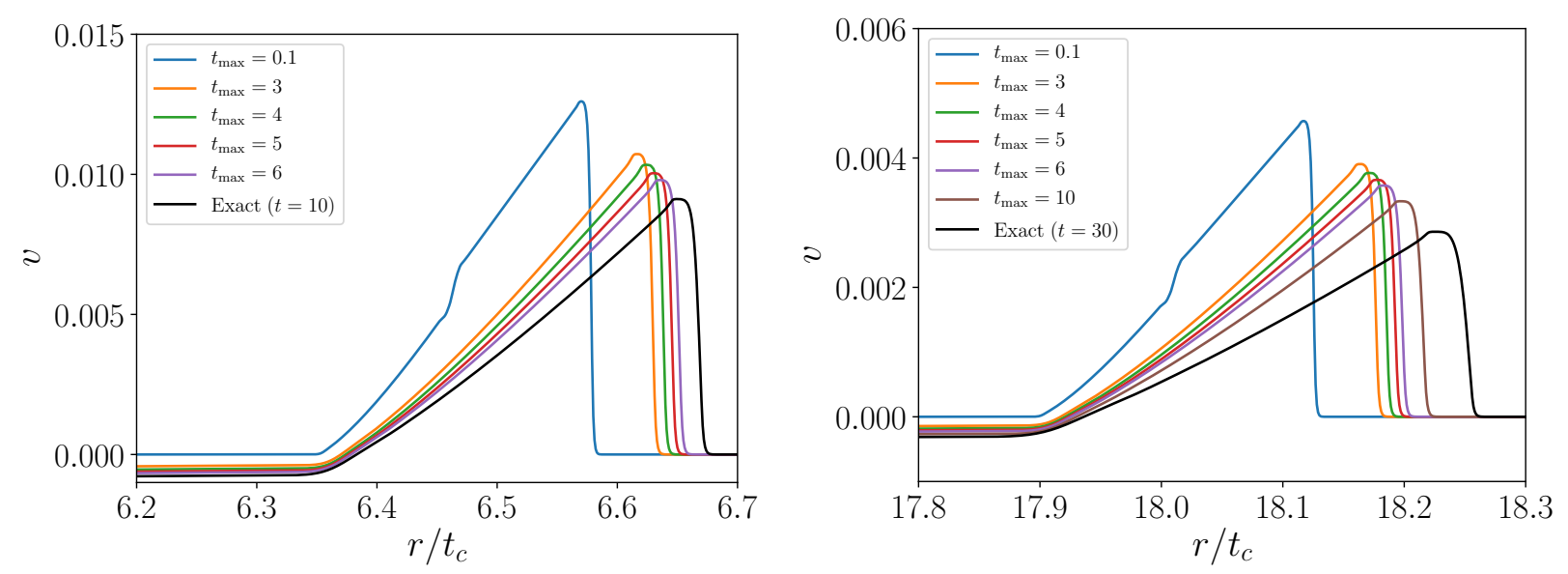

Figure 13: Test of the 1d simulation extrapolation. On the left, 10 time units after collision and, in the right, 30 time units after collision. We compare the effect of considering different maximum extrapolation times used in Eq. (45) with the exact numerical solution (black line). Notice that extrapolating the profile using any $t_{\text {max }}$ in the interval $[3,6]$ leads to similar results, which is rather close to the exact numerical result at short times. At later times (right), the extrapolation starts to fail, but the late time contribution to the final gravitational waves spectrum is smaller since it only affects very small bubbles.

Here the choice of $w(\vec{x})$ and $v^{i}(\vec{x})$ are the superpositions of the different bubble contributions. Notice that in linearizing the Euler equations, terms of $O\left(v^{2}\right)$ and $O\left(v\left(w / w_{0}-1\right)\right)$ are neglected but no terms of $O\left(\left(w / w_{0}-1\right)^{2}\right)$. Since the velocities point in different directions, they partially cancel out. If contributions of $n$ bubbles overlap, $v$ only grows as $\sqrt{n}$, while $\left(w / w_{0}-1\right)$ grows as $n$. Hence, in the regime of many bubbles overlapping, it makes a parametric difference if one superimposes $\left(w / w_{0}-1\right)$ or another quantity that is in leading order equivalent, e.g. $\log \left(w / w_{0}\right)$. Therefore, superimposing $\left(w / w_{0}-1\right)$ is the more consistent choice.

The second step involves taking the Fourier transform of each of the six components of $T^{i j}(\vec{x})$ using the FFTW3 package [39], obtaining the complex field $T^{i j}(\vec{k})$. The dual lattice sites of the compactified rectangular box are given by

$$
k_{i}=\sin \left(\frac{2 \pi i}{N}\right)
$$

Using this definition of $\vec{k}$, we calculate in the next step the projected components of the stressenergy tensor that appear in the equation of motion for $h$ (see Eq. (2)). We define $T_{+}(\vec{k})$ and $T_{\times}(\vec{k})$ as

$$
\begin{aligned}
& T_{+}(\vec{k})=\sum_{i, j} \frac{T^{i j}(\vec{k})}{\sqrt{2}}\left(\theta_{i}(\vec{k}) \theta_{j}(\vec{k})-\phi_{i}(\vec{k}) \phi_{j}(\vec{k})\right), \\
& T_{\times}(\vec{k})=\sum_{i, j} \frac{T^{i j}(\vec{k})}{\sqrt{2}}\left(\theta_{i}(\vec{k}) \phi_{j}(\vec{k})+\theta_{i}(\vec{k}) \phi_{j}(\vec{k})\right) .
\end{aligned}
$$

Here $\theta_{i}$ and $\phi_{i}$ are normalized vectors that are orthogonal to the momentum defined in Eq. (47).

The fourth step involves calculating the Fourier transform with respect to time defined by 
Eq. (5). Here, instead of using FFTW, we stack past time slices as

$$
T_{+, \times}(q, \vec{k}, t)=\sum_{t^{\prime}=t_{\text {init }}}^{t} e^{i q t^{\prime}} T_{+, \times}\left(t^{\prime}, \vec{k}\right),
$$

in order to save memory during the simulation run. A few comments are in order concerning the choice of $t_{\text {init }}$ and the definition of $k$. As mentioned in the main text, the typical $t_{\text {init }}$ is taken to be $\sim 10 / \beta$ time units after the nucleation of the first bubble, such that the GW from the sound shell does not get contaminated by IR effects related to GWs generated at the bubble size scale. The next point concerns the definition of $k$. Since $k$ is defined through the dispersion relation

$$
-k^{2}=\triangle=\nabla_{i} \nabla^{i}
$$

and the Laplacian operator needs to be invertible, we use

$$
\nabla_{i}=2 \frac{N}{L} \sin \left(\frac{\pi i}{N}\right)
$$

in contrast to (47).

The last step is to calculate the GW spectrum as defined in Eq. (14), taking the mean of $T_{+} T_{+}^{*}+T_{\times} T_{\times}^{*}$ in the lattice sites with the correct norm

$$
\Omega(q, t)=\left.C q^{3}\left\langle T_{+} T_{+}^{*}+T_{\times} T_{\times}^{*}\right\rangle\right|_{|\vec{k}|=q} .
$$

The constant $C$ is a normalization of the Fourier transform

$$
C=\Delta t^{2}\left(\frac{L^{3}}{N^{6}}\right)
$$

\section{The first collision of the fluid}

In this appendix we discuss how much the fluid profile is deformed at the first crossing. One might wonder what happens after enthalpy injection from bubble collision stops and how the fluid profile is deformed by the collision.

In order to test this, we perform a 1d fluid simulation of the type described in Section 2.2 and numerically evaluated according to Appendix A for collisions of deflagrations, hybrid and detonation initial profiles. We stress that our special concern with the first collision is that it is also the collision of the scalar field solitons. In subsequent fluid shockwave collisions there is no bubble wall involved. In that case, our model considers the linear approximation, justified in the small fluid velocity limit, in which the fluid profiles simply superimpose and cross each other.

We take as initial conditions for the two frontally colliding fluid profiles the exact moment in which the two (incoming and outgoing) solitons collide. It is important to consider this exact moment since the 1d fluid simulation no longer absorbs the enthalpy injection from the scalar field. Starting the collision simulation earlier makes the system start to lose energy earlier. The initial condition for deflagration, hybrid and detonations are respectively described by the black lines in the top, middle and bottom panels of Figure 14. In the left we show the velocity and in the right the enthalpy profile. Notice that in the case of deflagration and hybrid profiles, the fluid profiles (or part of them) already crossed each other when the scalar solitons collide. In that case, again, the linear approximation is used to justify that both waves pass each other without interacting. 
After some time, the spectral shape of the velocity and enthalpy profiles (for all three types of initial conditions) resembles the superposition of the initial profiles. Remarkably, the fluid profiles are not affected much after crossing each other. Notice that in the deflagration case there is a tail (also present in the single profile simulation), which results from the fact that the outgoing wave contains left- and right-moving contributions. Also the front part of the wave deforms: the peak at $\Delta t=0.3$ is slightly higher than at collision time. This ultimately leads to the enhancement of the kinetic energy $\left\langle w \gamma^{2} v^{2}\right\rangle_{1 \mathrm{~d}} / w_{\infty} \gtrsim \kappa \alpha$ discussed in the main text and in the following section.

Finally, we notice that a short time after collision and for all three profile types, the enthalpy in the region in between the two incoming and outgoing profile settles down to the same value as the innermost part of the two bubbles. Ultimately, this is a consequence of energy-momentum conservation.

\section{Normalization of the spectrum}

In Sec. 3.2 we presented the integrated spectrum $Q_{\text {int }}$ normalized by $\kappa \alpha,\left\langle w \gamma^{2} v^{2}\right\rangle_{1 \mathrm{~d}}$, and $\left\langle w \gamma^{2} v^{2}\right\rangle_{3 \mathrm{~d}}$. We found that the difference between $\kappa \alpha$ and $\left\langle w \gamma^{2} v^{2}\right\rangle_{1 \mathrm{~d}}$ is larger for smaller wall velocities. In this appendix we discuss this behavior in more detail.

Fig. 15 shows the time evolution of the fluid profile for $\alpha=0.0046$ and $\xi_{w}=0.32$. While $\Delta w=w-w_{0}$ decays relatively quickly, the maximal radial velocity $v$ almost keeps its original value for a while though the shell volume increases as $\propto r^{2}$ (see the first few gray lines in the right panel). This is the origin of the increase in the fluid kinetic energy in Fig. 16. In this figure we plot the time evolution of $\left\langle w \gamma^{2} v^{2}\right\rangle_{1 \mathrm{~d}}$ from $t=t_{c}$ to $7 t_{c}$. Note that $\left\langle w \gamma^{2} v^{2}\right\rangle_{1 \mathrm{~d}}\left(t=t_{c}\right)$ is equals to $(4 / 3) \kappa \alpha$, while we used $\left\langle w \gamma^{2} v^{2}\right\rangle_{1 \mathrm{~d}}\left(t=7 t_{c}\right)$ as the measure for the fluid kinetic energy in Sec. 3.2. The rearrangement effect from the decay into left- and right-moving waves after the energy injection from the Higgs wall terminates is stronger for small $\xi_{w}$ as seen from the top panel of Fig. 16. It is also seen that the kinetic energy decreases for $\xi_{w}$ close to the sound speed. This behavior seems typical for hybrid modes. As we decrease $\alpha$ (fixing $\xi_{w}$ ) the expansion mode becomes a deflagration, and the rearrangement effect is not as strong any more. This is why the data point $\alpha=0.0015$ and $\xi_{w}=0.6$ in Fig. 10 deviates from the gray line while that for $\alpha=0.0005$ and $\xi_{w}=0.6$ it is almost on top of it. As argued in Section 3.2, energy is still conserved and we checked this explicitly. We also plot the time evolution of $\left\langle w \gamma^{2} v^{2}\right\rangle_{3 \mathrm{~d}}$ in Fig. 17.

\section{E Fitting}

In this appendix we compare the data and the fitting function for the GW spectrum. Fig. 18 shows the data (blue) and fitting function (red) for $\alpha=0.0005,0.0015,0.0046,0.015,0.05$ (from top to bottom) and $\xi_{w}=0.32,0.36, \cdots, 0.8$ (from left to right) for the box size $L=40 \xi_{w} / \beta$ and grid $N=256$. The integration range is from $t=14 / \beta$ to $22 / \beta$. Fig. 19 is for $\alpha=0.0046$ (top) and 0.05 (bottom) and $\xi=0.32,0.36, \cdots, 0.8$ (from left to right) for the box size $L=80 \xi_{w} / \beta$ and grid $N=512$ with the same integration range. 

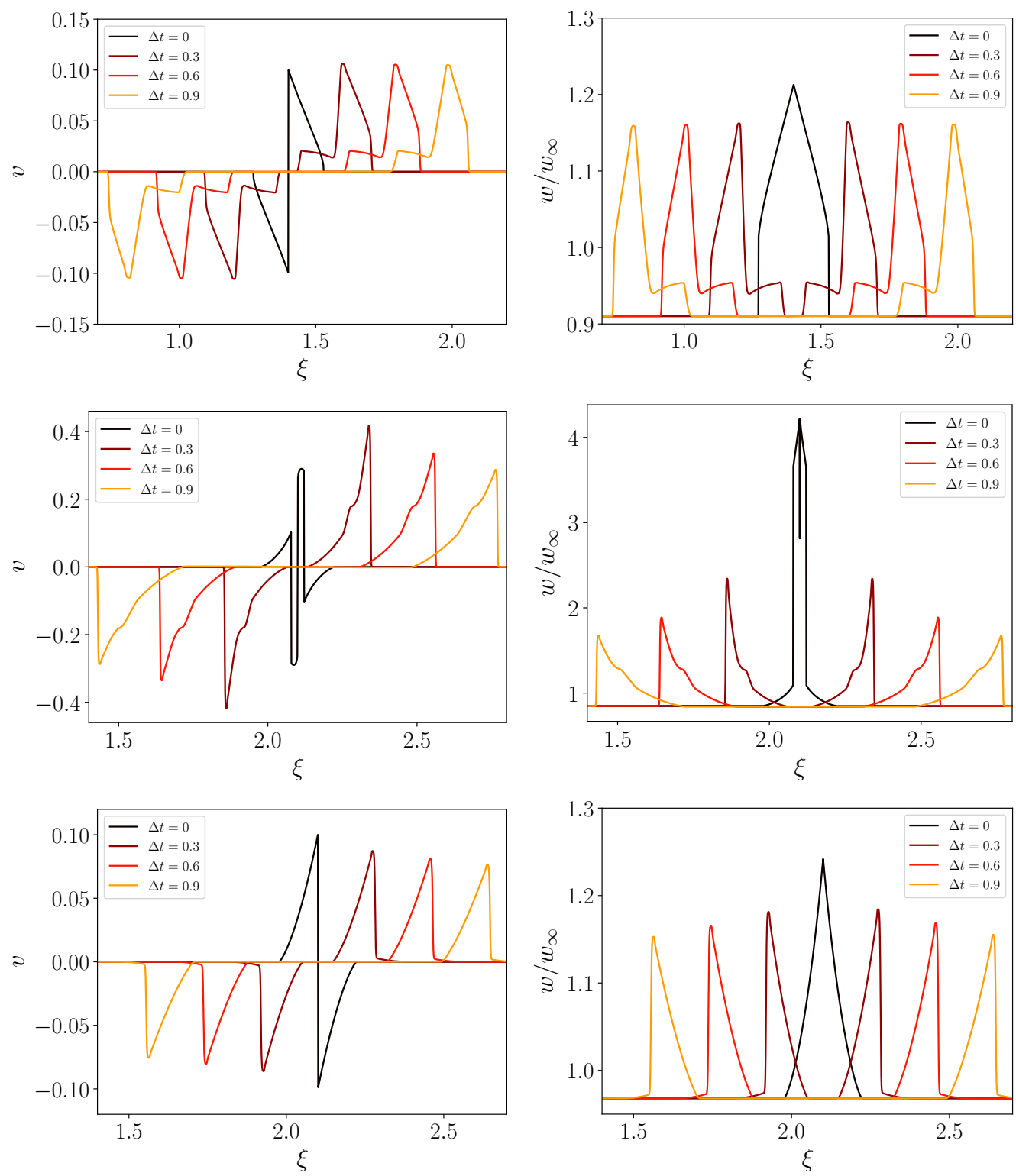

Figure 14: Effect of first fluid collision for deflagration (top), hybrid (middle) and detonation (bottom), in the left for the velocity and in the right for the enthalpy profile. We set the initial profile at the moment of the bubble wall collision. For all three kind of profiles, after the collision, the enthalpy value in the inner part between the two bubbles is the same as in the innermost part of a single bubble. 

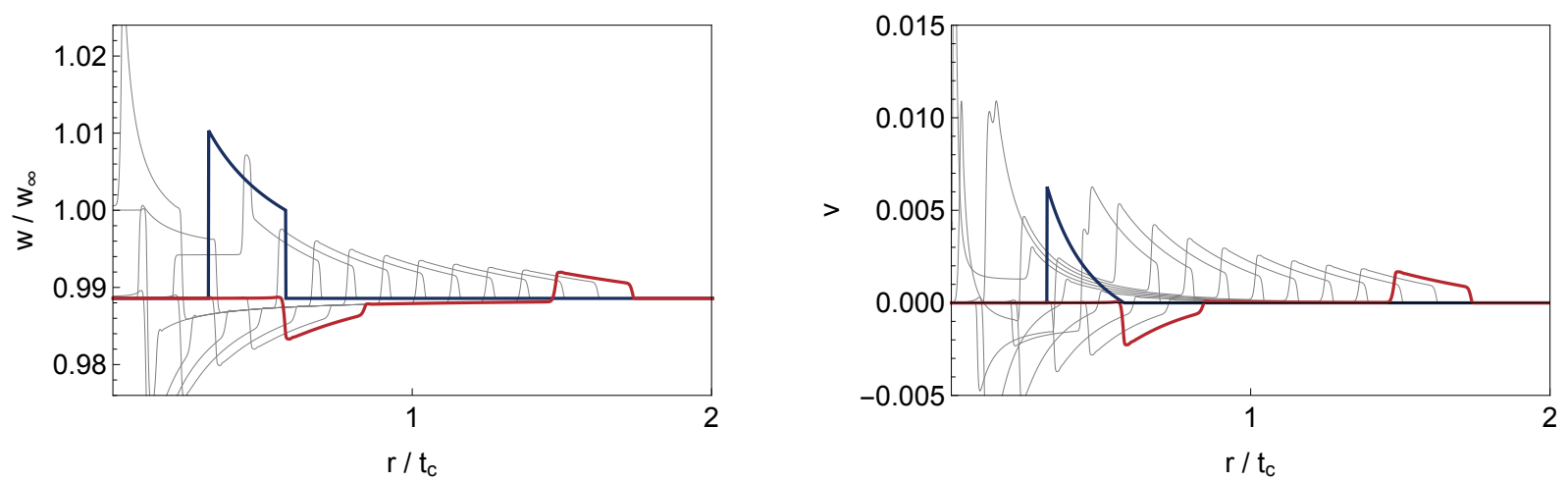

Figure 15: Time evolution of the fluid profile for $\alpha=0.0046$ and $\xi_{w}=0.32$ from $t=t_{c}$ (blue) to $2 t_{c}$ (red).
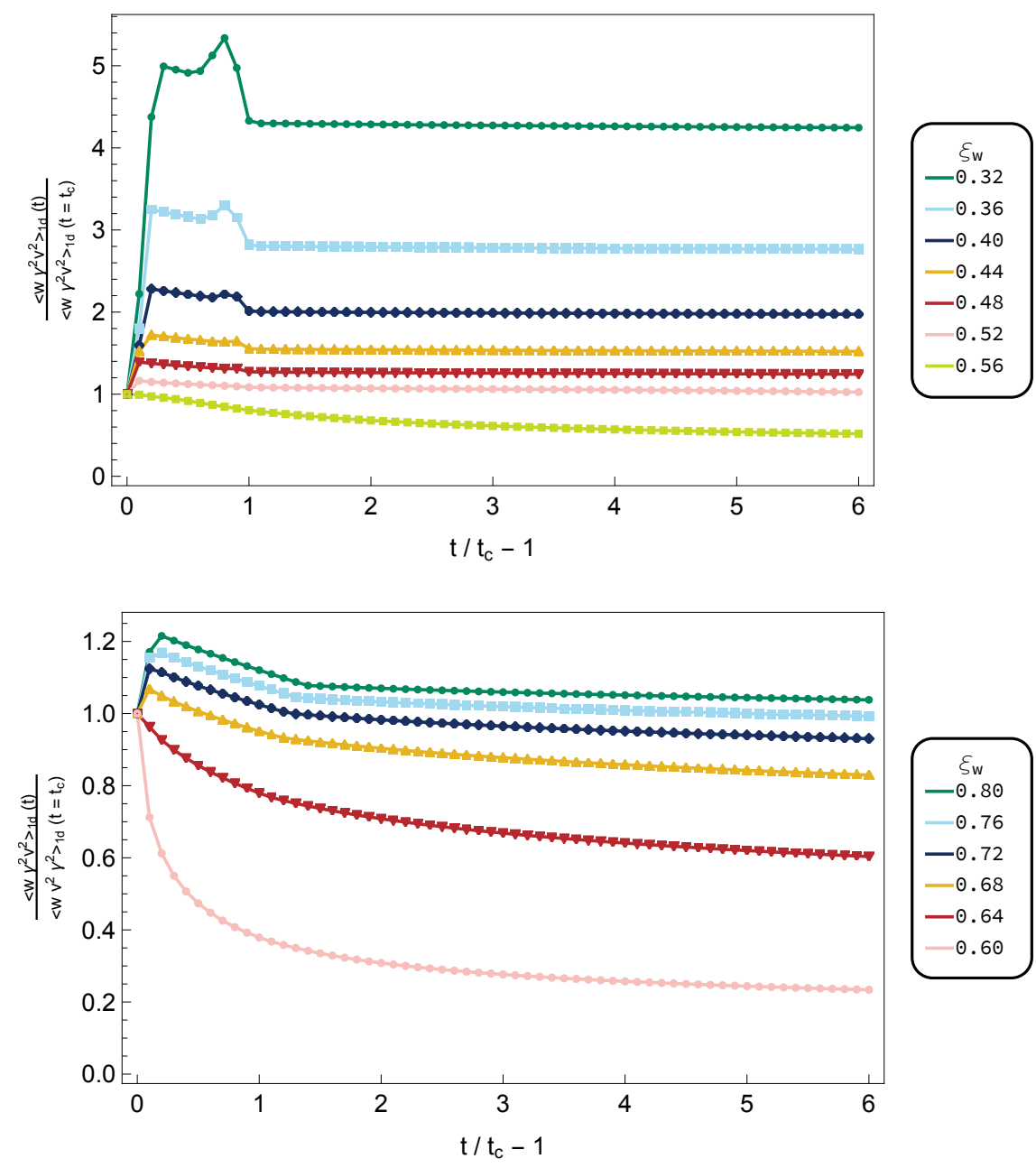

Figure 16: Time evolution of $\left\langle w \gamma^{2} v^{2}\right\rangle_{1 \mathrm{~d}}$ for small (top) and large (bottom) $\xi_{w}$. In this plot we fix $\alpha=0.0046$. Note that the normalization provided in the main text is $\left\langle w \gamma^{2} v^{2}\right\rangle_{1 \mathrm{~d}}\left(t=7 t_{c}\right)$ while in this plot we show its time evolution from $t=t_{c}$ to $7 t_{c}$. 

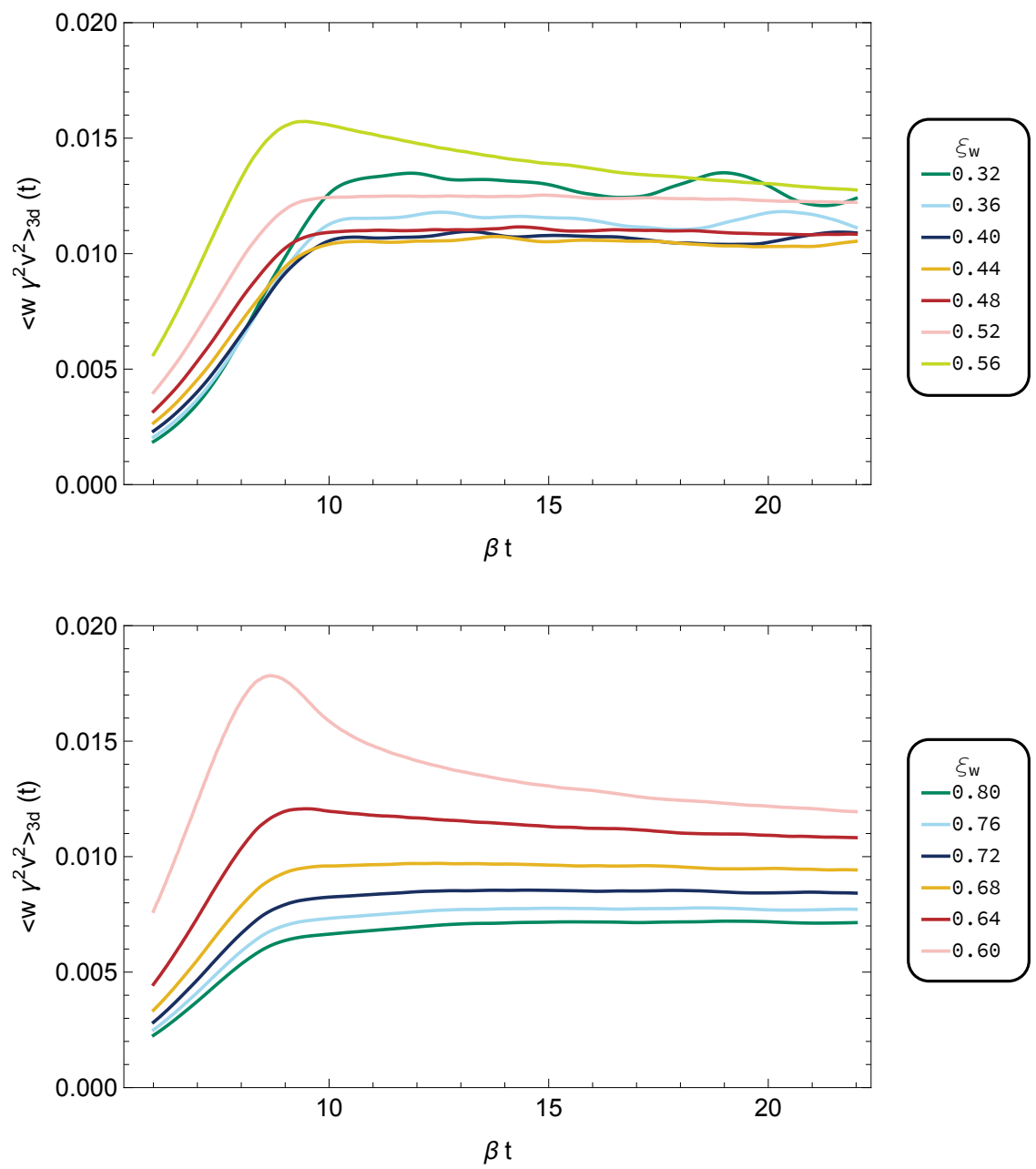

Figure 17: Time evolution of $\left\langle w \gamma^{2} v^{2}\right\rangle_{3 \mathrm{~d}}$ for small (top) and large (bottom) $\xi_{w}$. In this plot we fix $\alpha=0.0046$. 


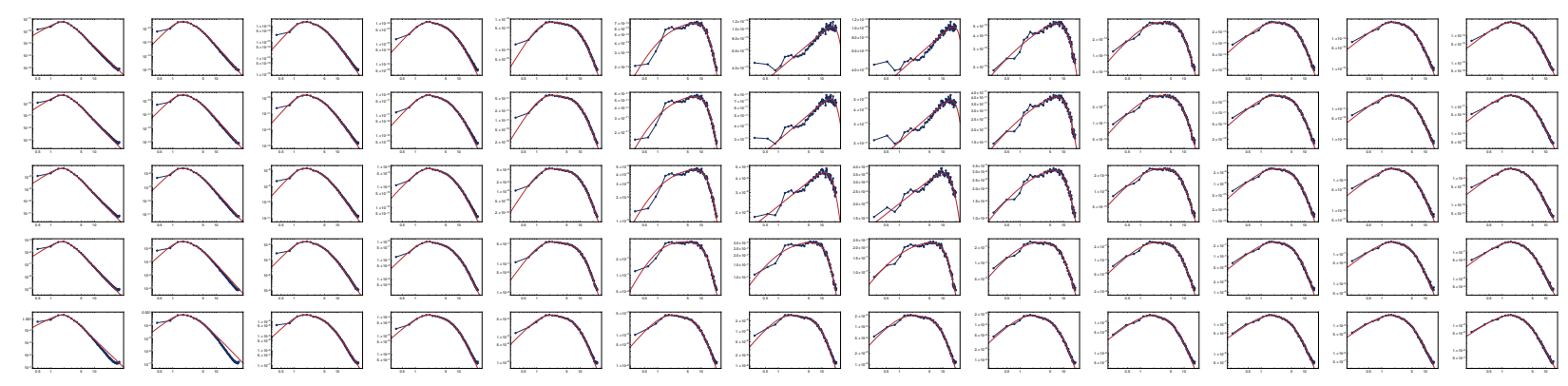

Figure 18: Data (blue) and fitting function (red) for $\alpha=0.0005,0.0015,0.0046,0.015$ and 0.05 (from top to bottom) and $\xi_{w}=0.32,0.36, \cdots, 0.8$ (from left to right). The horizontal and vertical axes are $q / \beta$ and $Q^{\prime}$, respectively. We use box size $L=40 \xi_{w} / \beta$ and grid $N=256$.

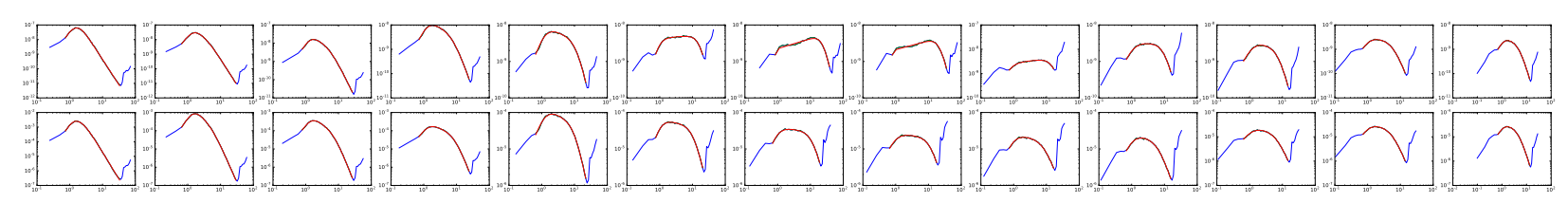

Figure 19: Same as Fig. 18 except that we use $L=80 \xi_{w} / \beta$ and $N=512$. 


\section{References}

[1] LIGO Scientific, Virgo Collaboration, B. Abbott et al., "Observation of Gravitational Waves from a Binary Black Hole Merger," Phys. Rev. Lett. 116 no. 6, (2016) 061102, arXiv:1602.03837 [gr-qc].

[2] LIGO Scientific, Virgo Collaboration, B. P. Abbott et al., "GW151226: Observation of Gravitational Waves from a 22-Solar-Mass Binary Black Hole Coalescence," Phys. Rev. Lett. 116 no. 24, (2016) 241103, arXiv:1606.04855 [gr-qc].

[3] LIGO Scientific, Virgo Collaboration, B. Abbott et al., "GW170817: Observation of Gravitational Waves from a Binary Neutron Star Inspiral," Phys. Rev. Lett. 119 no. 16, (2017) 161101, arXiv:1710.05832 [gr-qc].

[4] eLISA Collaboration, P. A. Seoane et al., "The Gravitational Universe," arXiv:1305.5720 [astro-ph.CO].

[5] P. Amaro-Seoane, H. Audley, S. Babak, J. Baker, E. Barausse, P. Bender, E. Berti, P. Binetruy, M. Born, D. Bortoluzzi, J. Camp, C. Caprini, V. Cardoso, M. Colpi, J. Conklin, N. Cornish, C. Cutler, K. Danzmann, R. Dolesi, L. Ferraioli, V. Ferroni, E. Fitzsimons, J. Gair, L. G. Bote, D. Giardini, F. Gibert, C. Grimani, H. Halloin, G. Heinzel, T. Hertog, M. Hewitson, K. Holley-Bockelmann, D. Hollington, M. Hueller, H. Inchauspe, P. Jetzer, N. Karnesis, C. Killow, A. Klein, B. Klipstein, N. Korsakova, S. L. Larson, J. Livas, I. Lloro, N. Man, D. Mance, J. Martino, I. Mateos, K. McKenzie, S. T. McWilliams, C. Miller, G. Mueller, G. Nardini, G. Nelemans, M. Nofrarias, A. Petiteau, P. Pivato, E. Plagnol, E. Porter, J. Reiche, D. Robertson, N. Robertson, E. Rossi, G. Russano, B. Schutz, A. Sesana, D. Shoemaker, J. Slutsky, C. F. Sopuerta, T. Sumner, N. Tamanini, I. Thorpe, M. Troebs, M. Vallisneri, A. Vecchio, D. Vetrugno, S. Vitale, M. Volonteri, G. Wanner, H. Ward, P. Wass, W. Weber, J. Ziemer, and P. Zweifel, "Laser interferometer space antenna," 2017.

[6] K. Kajantie, M. Laine, K. Rummukainen, and M. E. Shaposhnikov, "Is there a hot electroweak phase transition at $\mathrm{m}(\mathrm{H})$ larger or equal to $\mathrm{m}(\mathrm{W})$ ?," Phys. Rev. Lett. 77 (1996) 2887-2890, arXiv:hep-ph/9605288.

[7] M. Gurtler, E.-M. Ilgenfritz, and A. Schiller, "Where the electroweak phase transition ends," Phys. Rev. D 56 (1997) 3888-3895, arXiv:hep-lat/9704013.

[8] F. Csikor, Z. Fodor, and J. Heitger, "Endpoint of the hot electroweak phase transition," Phys. Rev. Lett. 82 (1999) 21-24, arXiv:hep-ph/9809291.

[9] V. A. Kuzmin, V. A. Rubakov, and M. E. Shaposhnikov, "On the Anomalous Electroweak Baryon Number Nonconservation in the Early Universe," Phys. Lett. 155B (1985) 36.

[10] A. G. Cohen, D. Kaplan, and A. Nelson, "Progress in electroweak baryogenesis," Ann. Rev. Nucl. Part. Sci. 43 (1993) 27-70, arXiv:hep-ph/9302210.

[11] V. Rubakov and M. Shaposhnikov, "Electroweak baryon number nonconservation in the early universe and in high-energy collisions," Usp. Fiz. Nauk 166 (1996) 493-537, arXiv:hep-ph/9603208.

[12] A. Riotto and M. Trodden, "Recent progress in baryogenesis," Ann. Rev. Nucl. Part. Sci. 49 (1999) 35-75, arXiv:hep-ph/9901362.

[13] D. E. Morrissey and M. J. Ramsey-Musolf, "Electroweak baryogenesis," New J. Phys. 14 (2012) 125003, arXiv:1206.2942 [hep-ph].

[14] S. R. Coleman, "The Fate of the False Vacuum. 1. Semiclassical Theory," Phys. Rev. D 15 (1977) 2929-2936. [Erratum: Phys.Rev.D 16, 1248 (1977)].

[15] A. D. Linde, "Fate of the False Vacuum at Finite Temperature: Theory and Applications," Phys. Lett. B 100 (1981) 37-40.

[16] P. J. Steinhardt, "Relativistic Detonation Waves and Bubble Growth in False Vacuum Decay," Phys. Rev. D 25 (1982) 2074. 
[17] E. Witten, "Cosmic Separation of Phases," Phys. Rev. D30 (1984) 272-285.

[18] J. Ellis, M. Lewicki, J. M. No, and V. Vaskonen, "Gravitational wave energy budget in strongly supercooled phase transitions," JCAP 1906 no. 06, (2019) 024, arXiv:1903.09642 [hep-ph].

[19] D. Bodeker and G. D. Moore, "Electroweak Bubble Wall Speed Limit," JCAP 1705 no. 05, (2017) 025, arXiv:1703.08215 [hep-ph].

[20] D. Bodeker and G. D. Moore, "Can electroweak bubble walls run away?," JCAP 0905 (2009) 009, arXiv:0903.4099 [hep-ph].

[21] S. Hche, J. Kozaczuk, A. J. Long, J. Turner, and Y. Wang, "Towards an all-orders calculation of the electroweak bubble wall velocity," arXiv:2007.10343 [hep-ph].

[22] F. Giese, T. Konstandin, and J. van de Vis, "Model-independent energy budget of cosmological first-order phase transitions: A sound argument to go beyond the bag model," JCAP 07 no. 07, (2020) 057, arXiv:2004.06995 [astro-ph.CO].

[23] M. Hindmarsh, S. J. Huber, K. Rummukainen, and D. J. Weir, "Gravitational waves from the sound of a first order phase transition," Phys. Rev. Lett. 112 (2014) 041301, arXiv:1304.2433 [hep-ph].

[24] M. Hindmarsh, S. J. Huber, K. Rummukainen, and D. J. Weir, "Numerical simulations of acoustically generated gravitational waves at a first order phase transition," Phys. Rev. D92 no. 12, (2015) 123009, arXiv:1504.03291 [astro-ph.CO].

[25] M. Hindmarsh, S. J. Huber, K. Rummukainen, and D. J. Weir, "Shape of the acoustic gravitational wave power spectrum from a first order phase transition," arXiv:1704.05871 [astro-ph.CO].

[26] J. R. Espinosa, T. Konstandin, J. M. No, and G. Servant, "Energy Budget of Cosmological First-order Phase Transitions," JCAP 1006 (2010) 028, arXiv:1004.4187 [hep-ph].

[27] R. Jinno, H. Seong, M. Takimoto, and C. M. Um, "Gravitational waves from first-order phase transitions: Ultra-supercooled transitions and the fate of relativistic shocks," JCAP 10 (2019) 033, arXiv:1905.00899 [astro-ph.CO].

[28] A. Kurganov and E. Tadmor, "New high-resolution central schemes for nonlinear conservation laws and convection-diffusion equations," Journal of Computational Physics 160 no. 1, (2000) 241 - 282. http://www.sciencedirect.com/science/article/pii/S0021999100964593.

[29] M. Lewicki and V. Vaskonen, "Gravitational wave spectra from strongly supercooled phase transitions," arXiv:2007.04967 [astro-ph.CO].

[30] C. Caprini et al., "Detecting gravitational waves from cosmological phase transitions with LISA: an update," JCAP 03 (2020) 024, arXiv:1910.13125 [astro-ph.CO].

[31] C. R. McKee and S. A. Colgate, "Relativistic Shock Hydrodynamics," apj 181 (May, 1973) 903-938.

[32] R. Jinno and M. Takimoto, "Gravitational waves from bubble dynamics: Beyond the Envelope," JCAP 1901 (2019) 060, arXiv:1707.03111 [hep-ph].

[33] T. Konstandin, "Gravitational radiation from a bulk flow model," JCAP 1803 no. 03, (2018) 047, arXiv:1712.06869 [astro-ph.CO].

[34] M. Hindmarsh, "Sound shell model for acoustic gravitational wave production at a first-order phase transition in the early Universe," Phys. Rev. Lett. 120 no. 7, (2018) 071301, arXiv:1608.04735 [astro-ph.CO].

[35] M. Hindmarsh and M. Hijazi, "Gravitational waves from first order cosmological phase transitions in the Sound Shell Model," JCAP 1912 (2019) 062, arXiv:1909.10040 [astro-ph.CO].

[36] F. Giese, T. Konstandin, K. Schmitz, and J. van de Vis, "To appear,". 
[37] P. D. Lax, "Weak solutions of nonlinear hyperbolic equations and their numerical computation," Communications on Pure and Applied Mathematics 7 no. 1, (1954) 159-193, https://onlinelibrary.wiley.com/doi/pdf/10.1002/cpa.3160070112. https://onlinelibrary.wiley.com/doi/abs/10.1002/cpa.3160070112.

[38] K. Friedrichs, "Symmetric hyperbolic linear differential equations," Commun. Pure Appl. Math. 7 (1954) 345-392.

[39] M. Frigo and S. Johnson, "The Design and Implementation of FFTW3," IEEE Proc. 93 no. 2, (2005) $216-231$. 